\title{
INNER IDEALS IN QUADRATIC JORDAN ALGEBRAS
}

\author{
BY \\ KEVIN MCCRIMMON
}

\begin{abstract}
The inner ideals play a role in the theory of quadratic Jordan algebras analogous to that played by the one-sided ideals in the theory of associative algebras. In particular, the Jordan algebras with descending chain condition on inner ideals are intimately related to the Artinian associative algebras. In this paper we will completely characterize all inner ideals in the semisimple Jordan algebras with descending chain condition. It is well known that any left or right ideal $\mathfrak{B}$ in a semisimple Artinian $\mathfrak{U}$ is determined by an idempotent, $\mathfrak{B}=\mathfrak{U} f$ or $\mathfrak{B}=e \mathfrak{A}$. We show that any inner ideal in the quadratic Jordan algebra $\mathfrak{A}^{+}$has the form $\mathfrak{B}=e \mathfrak{A} f$, and if $\mathscr{A}$ has involution * the inner ideals of the Jordan algebra $\mathfrak{H}\left(\mathfrak{A},{ }^{*}\right)$ of *-symmetric elements are "usually" of the form $\mathfrak{B}=e^{*} \mathfrak{h} e$. We also characterize the inner ideals in the Jordan algebras $\mathfrak{I}(Q, c)$ or $\mathfrak{I}(N, c)$ determined by a quadratic or cubic form.
\end{abstract}

The Second Structure Theorem says that a simple, unital Jordan algebra with d.c.c. on principal inner ideals belongs to one of six types. We begin by showing conversely that any algebra of such type has the d.c.c. on principal inner ideals, and characterizing the inner ideals in those types of capacity $\geqq 3$. The proof makes detailed use of the structure theory, in particular the Coordinatization Theorem. Then we re-investigate the six types of simple algebras case by case, characterizing their inner ideals by more geometric methods.

1. Preliminaries. We recall some of the basic properties relating to inner ideals. Throughout we work with unital quadratic Jordan algebras (see [2], [3]) over an arbitrary commutative, associative ring of scalars $\Phi$. Thus $\Im$ is equipped with a cubic composition $(x, y) \rightarrow U_{x} y$ which satisfies (among other axioms) the Fundamental Formula

$$
U_{U(x) y}=U_{x} U_{y} U_{x}
$$

One obtains a Jordan algebra $\mathfrak{A}^{+}$from an associative algebra $\mathfrak{A}$ by taking $U_{x} y$ $=x y x$. An inner ideal is a subspace $\mathfrak{B}$ of $\mathfrak{\Im}$ such that $U_{\mathfrak{B} \mathfrak{B}} \subset \mathfrak{B}$; the Fundamental Formula shows that if $b$ is any element of $\Im$ then $U_{b} \Im$ is an inner ideal, called the principal inner ideal determined by $b$. An important example is when $b=e$ is idempotent, $e^{2}=e$, in which case $U_{e} \mathfrak{\Im}$ is just the Peirce 1 -space $\mathfrak{\Im}_{1}(e)$.

Given an invertible element $u \in \mathfrak{F}$ we can form a new Jordan algebra, the $u$-isotope $\mathfrak{\Im}^{(u)}$, with unit $1^{(u)}=u^{-1}$ and $U$-operator $U_{x}^{(u)}=U_{x} U_{u}$. It is important that $\mathfrak{I}$ and its isotopes have the same inner ideals.

Received by the editors August 17, 1970.

AMS 1969 subject classifications. Primary 1740.

Key words and phrases. Quadratic Jordan algebra, inner ideal.

Copyright (C) 1971, American Mathematical Society 
We recall [2], [3] the basic

Minimal InNer Ideal TheORem. If $\mathfrak{B}$ is a minimal inner ideal in a Jordan algebra $\Im$ then $\mathfrak{B}$ has one of the following forms:

I. $\mathfrak{B}=\Phi z$ for $z$ trivial, $U_{z}=0$;

II. $\mathfrak{B}=U_{b} \Im$ for all $b \neq 0$ in $\mathfrak{B}$ but $U_{\mathfrak{B}} \mathfrak{B}=\mathfrak{B}^{2}=0$;

III. $\mathfrak{B}=U_{e} \mathfrak{I}$ for e a completely primitive idempotent.

In case II there is an isotope $\mathfrak{\Im}^{(u)}$ in which $\mathfrak{B}$ is of type III, $\mathfrak{B}=U_{e^{(u)}}^{(u)} \mathfrak{\Im}^{(u)}$ for $e^{(u)}$ completely primitive in $\Im^{(u)}$.

An element $x$ is regular (in the sense of von Neumann) if $x=U_{x} y$ for some $y$; an algebra is regular if all its elements are. This will prove a more useful condition than requiring $\Im$ to be semisimple in the sense that its Jacobson radical is zero. For algebras satisfying the descending chain condition (or minimal condition) on inner ideals the following are equivalent (see [4]):

(i) $\mathfrak{\Im}$ is semisimple.

(ii) $\Im$ is regular.

(iii) $\Im$ contains no trivial elements $z \neq 0$ with $U_{z}=0$.

It is well known that the same equivalence holds in Artinian associative algebras (with $U_{z} \mathfrak{A}=z \mathfrak{A} z$ ).

LEMMA 1. Let $\mathfrak{\Im}$ contain no trivial elements. If $\mathfrak{B}$ is a principal inner ideal minimal among all principal inner ideals, then $\mathfrak{B}$ is minimal among all inner ideals. If $\mathfrak{\Im}$ has minimum condition on principal inner ideals then every nonzero inner ideal contains a minimal inner ideal.

Proof. Let $\mathfrak{B}$ be minimal among all principal inner ideals but suppose $\mathfrak{B}>\mathfrak{C}>0$ for some (naturally nonprincipal) inner ideal $\mathfrak{\complement}$. If $c$ is any nonzero element of $\mathfrak{c}$ we have $U_{c} \mathfrak{\Im} \subset \mathbb{C}$ since $\mathbb{C}$ is inner; $U_{c} \mathfrak{F} \neq 0$ since $c \neq 0$ implies $c$ is not trivial by our hypothesis. But then $U_{c} \mathfrak{I}$ is a nonzero principal inner ideal strictly smaller than $\mathfrak{B}, U_{c} \mathfrak{\Im} \subset \mathfrak{C}<\mathfrak{B}$, contradicting the minimality of $\mathfrak{B}$.

Now suppose $\mathfrak{\Im}$ has d.c.c. on principal inner ideals, and let $\mathfrak{B}$ be any nonzero inner ideal. Then $\mathfrak{B}$ contains nonzero principal inner ideals $U_{b} \Im \neq 0$ (since $b \neq 0$ cannot be trivial), so it contains a minimal principal inner ideal $\mathbb{E}$ by the d.c.c., and by the above $\mathbb{c}$ is actually a minimal inner ideal.

The structure group of $\mathfrak{I}$ is the set of bijective linear transformation $T$ on $\mathfrak{I}$ for which there exists a bijective $T^{*}$ satisfying

$$
U_{T x}=T U_{x} T^{*}
$$

for all $x \in \mathfrak{\Im}$. From this it is clear that any element $T$ of the structure group preserves inner ideals: $T \mathfrak{B}$ is inner if $\mathfrak{B}$ is inner. The structure group can also be described as the isotopies of $\mathfrak{\Im}$, the isomorphisms of $\mathfrak{\Im}$ with its isotopes.

The First Structure Theorem for quadratic Jordan algebras with d.c.c. on principal inner ideals [2], [3] says that every semisimple such algebra is a direct 
sum of a finite number of simple ideals. The Second Structure Theorem says the simple ideals are isotopic to one of the following:

I. a Jordan division algebra;

II. a Jordan algebra $\Im(Q, c)$ determined by a nondegenerate quadratic form;

II'. a split algebra $\Im\left(Q_{0}, \Phi_{0}\right)$ for $\Phi_{0}$ an ample subspace of $\Phi$;

III. $\Delta_{n}^{+}$for $\Delta$ an associative division algebra, $n \geqq 2$;

IV. $\mathfrak{S}\left(\Delta_{n}, \Delta_{0}\right)$ for $n \geqq 2, \Delta$ an associative division algebra with involution (nontrivial if $n=2)$ and $\Delta_{0}$ an ample subspace;

V. $\mathfrak{S}\left(\mathfrak{Q}_{n}, \Phi\right)$ for $\mathfrak{Q}=\Phi_{2}$ a split quaternion algebra over its center $\Phi$;

VI. $\mathfrak{S}\left(\mathfrak{\complement}_{3}\right)$ for $\mathfrak{C}$ a Cayley algebra over its center.

These are the algebras whose inner ideals we will investigate. We get rid of case I right away by means of

Proposition 1. A Jordan algebra is a division algebra if and only if it is not trivial and has no proper inner ideals.

Proof. If $\mathfrak{J}$ is a division algebra, each element $b \neq 0$ is invertible, thus $U_{b}$ is invertible, so if $\mathfrak{B}$ is an inner ideal and $b \neq 0$ is in $\mathfrak{B}$ we have $\mathfrak{B} \supset U_{b} \mathfrak{\Im}=\mathfrak{\Im}$. Conversely, if $\mathfrak{\Im}$ has no proper inner ideals we must have $U_{b} \mathfrak{I}=\mathfrak{I}$ for all $b$, otherwise $U_{b} \mathfrak{\Im}=0$ and $\Phi b$ is an inner ideal; but $\Phi b=\mathfrak{J}$ would imply $\mathfrak{I}$ is trivial, $U_{\mathfrak{J}} \mathfrak{J}=0$. As soon as one $U_{b}$ is surjective the algebra $\Im$ necessarily has a unit (see [5]), therefore 1 is in the range of all $U_{b}(b \neq 0)$ and so $U_{b}$ and $b$ are invertible.

Notice that this corresponds to the fact that an associative algebra is a division algebra if and only if it is not trivial and contains no proper one-sided ideals.

2. The d.c.c. for principal inner ideals. Here we want to establish the d.c.c. at least for principal inner ideals in the algebras in which we are interested. For finitedimensional situations we can say more.

Proposition 2. If $\Im$ is a regular Jordan algebra then each inner ideal is invariant under the centroid $\Gamma(\Im)$. In particular, if $\mathfrak{\Im}$ is regular and finite dimensional over a field $\Phi$ then $\mathfrak{\Im}$ has the d.c.c. and a.c.c. on all inner ideals.

Proof. Let $\mathfrak{B}$ be an inner ideal in $\mathfrak{\Im}, b \in \mathfrak{B}$, and $T \in \Gamma(\mathfrak{\Im})$. Then $b=U_{b} a$ for some $a \in \mathfrak{F}$ by regularity, so $T b=T\left(U_{b} a\right)=U_{b}(T a) \in U_{b} \mathfrak{F} \subset \mathfrak{B}$. This shows $\mathfrak{B}$ is invariant under $\Gamma(\Im)$. If $\Im$ is an algebra over $\Phi, \Phi$ is contained in the centroid and so all inner ideals are $\Phi$-subspaces. The rest is clear.

COROLlaRY. If $\mathfrak{I}=\mathfrak{S}\left(\mathfrak{D}_{n}, \gamma\right)$ for $\mathfrak{D}$ a composition algebra over a field $\Phi$ then $\mathfrak{I}$ has the a.c.c. and d.c.c. on all inner ideals.

Proof. $\mathfrak{D}$ has dimension $1,2,4$, or 8 over $\Phi$, so $\mathfrak{\Im}$ is finite dimensional. The regularity of such $\mathfrak{\Im}$ is well known.

COROLlaRY. If $\mathfrak{\Im}=\Im(Q, c)$ for $Q$ a nondegenerate quadratic form on a finitedimensional vector space $\mathfrak{X}$ over a field $\Phi$, then $\mathfrak{\Im}$ has a.c.c. and d.c.c. on all inner ideals. 
Proposition 3. If $\mathfrak{A}$ is a regular associative algebra with involution then $\mathfrak{S}\left(\mathfrak{A},{ }^{*}\right)$ is regular; indeed,

$$
x^{*} \mathfrak{A} x \cap \mathfrak{S}=x^{*} \mathfrak{S} x \quad\left(\mathfrak{S}=\mathfrak{S}\left(\mathfrak{A},{ }^{*}\right)\right)
$$

for any $x$ in $\mathfrak{A}$.

Proof. If $x=x^{*}$ belongs to $\mathfrak{S}$ then by regularity of $\mathfrak{A} x=x a x$ for some $a \in \mathfrak{A}$. We need to find a symmetric element $h$ which serves just as well. Now $x=x^{*} a x$ $=(x a x)^{*} a x=x a^{*} x a x=x h x$ where $h=a^{*} x a \in \mathfrak{H}$ is symmetric (if $\frac{1}{2} \in \Phi$ we could more easily set $\left.h=\frac{1}{2}\left(a+a^{*}\right)\right)$. This shows $\mathfrak{S}$ is regular.

For arbitrary $x \in \mathfrak{A}$, if $x^{*}$ ax belongs to $\mathfrak{S E}$ then by regularity of $\mathfrak{S}$ there is $h \in \mathfrak{S}$ with $x^{*} a x=\left(x^{*} a x\right) h\left(x^{*} a x\right)=\left(x^{*} a x\right)^{*} h\left(x^{*} a x\right)=x^{*} a^{*} x h x^{*} a x=x^{*} k x$ for $k=a^{*} x h x^{*} a$ $\in \mathfrak{F}$. This shows $x^{*} \mathfrak{A} x \cap \mathfrak{S C} \subset x^{*} \mathfrak{S} x$; the reverse inclusion is obvious.

COROllaRY. Any principal inner ideal $b \mathfrak{S} b$ in $\mathfrak{S}\left(\mathfrak{A},{ }^{*}\right)$ for $\mathfrak{A}$ regular is the intersection of $\mathfrak{S}$ with the principal inner ideal $b \mathfrak{A} b$ in $\mathfrak{A}$. If $\mathfrak{A}$ has the a.c.c. or d.c.c. on principal inner ideals then so does $\mathfrak{S}\left(\mathfrak{A},{ }^{*}\right)$.

Proof. If $b^{*}=b$ is symmetric then $b \mathfrak{S}_{\mathfrak{C}} b=b \mathfrak{A} b \cap \mathfrak{S}_{\mathfrak{S}}$ by the above. If $b_{1} \mathfrak{S}_{\mathfrak{E}} b_{1}$, $b_{2} \mathfrak{S}_{\mathfrak{C}} b_{2}, \ldots$ is an ascending or descending sequence of principal inner ideals in $\mathfrak{S}$ then $b_{1} \mathfrak{A} b_{1}, b_{2} \mathfrak{A} b_{2}, \ldots$ is also increasing or decreasing in $\mathfrak{A}$ by regularity (if $b \mathfrak{S e} b \subset c \mathfrak{F} c$ then $b \in b \mathfrak{S c} b \subset c \mathfrak{S c} c \subset c \mathfrak{A} c$ implies $b \mathfrak{A} b \subset c \mathfrak{A} c$ ), and if this latter sequence in $\mathfrak{A}$ is constant after some point then so is the sequence of $b_{i} \mathfrak{S} b_{i}=b_{i} \mathfrak{A} b_{i} \cap \mathfrak{S}$.

If $\mathfrak{A}$ is regular and has the a.c.c. or d.c.c. on left and right ideals then it also has it on principal inner ideals, since $b \mathfrak{A} b=b \mathfrak{A} \cap \mathfrak{A} b$ by regularity $(x=b a=c b \Rightarrow x$ $=x y x=b a y c b)$ and if $b \mathfrak{A} b \subset c \mathfrak{A} c$ then $b \in c \mathfrak{A} c$ so $b \mathfrak{A} \subset c \mathfrak{A}$ and $\mathfrak{A} b \subset \mathfrak{A} c$. Thus from the previous corollary we obtain

Proposition 4. If $\mathfrak{A}$ is a regular Artinian algebra with involution then $\mathfrak{S}\left(\mathfrak{A},{ }^{*}\right)$ is regular and has the a.c.c. and d.c.c. on principal inner ideals.

A particular example of an algebra with involution is $\widetilde{\mathfrak{A}}=\mathfrak{A} \oplus \mathfrak{H}^{0}$ under the exchange involution. Here $\mathfrak{S}\left(\tilde{\mathfrak{A}},{ }^{*}\right)$ is Jordan-isomorphic to $\mathfrak{A}^{+}$, so as a special case of Proposition 4 we see

Proposition 5. If $\mathfrak{A}$ is a regular Artinian algebra then $\mathfrak{A}^{+}$has the ascending and descending chain conditions on principal inner ideals.

Some of our simple Jordan algebras do not quite have the nice form $\mathfrak{S}\left(\mathfrak{A},{ }^{*}\right)$ or $\Im(Q, c)$, but rather are ample outer ideals in such standard examples. We do not have to establish regularity or the chain conditions separately for these outer ideals-they are inherited from the larger algebra.

Proposition 6. If $\mathfrak{\Re}$ is an outer ideal in a Jordan algebra $\mathfrak{\Im}$ then $\mathfrak{\Re}$ is regular if $\Im$ is. In this case

$$
\mathfrak{\Re} \cap U_{x} \mathfrak{\Im}=U_{x} \mathfrak{\AA} \quad(x \in \mathfrak{S})
$$

and $\mathfrak{\Re}$ has the a.c.c. or d.c.c. on principal inner ideals if $\mathfrak{\Im}$ does. 
Proof. For regularity, given $x \in \mathfrak{N}$ we have $x=U_{x} y$ for some $y \in \mathfrak{I}$ if $\mathfrak{I}$ is regular; in fact, we can assume $y=U_{y} x$ (replacing $y$ by $y^{\prime}=U_{y} x$ if necessary), so $y \in U_{\mathfrak{I}} \mathfrak{T} \subset \mathfrak{\Re}$ by definition of an outer ideal. Thus $\Omega$ inherits regularity. In this case, if $y \in \mathfrak{R} \cap U_{x} \mathfrak{I}$ then $y \in \mathfrak{R}$ has $y=U_{y} k$ for $k \in \mathfrak{R}$ since $\mathfrak{R}$ is regular, so $y=U_{y} k \in U_{U(x) \mathfrak{I}} k$ $=U_{x} U_{\mathfrak{I}} U_{x} k \subset U_{x} \mathfrak{\Re}$ since $\mathfrak{\Re}$ is outer. The reverse inclusion $U_{x} \mathfrak{T} \subset \mathfrak{\Re} \cap U_{x} \mathfrak{I}$ is clear. Also in this case, $b, c \in \mathfrak{N}$ have $U_{b} \mathfrak{T} \subset U_{c} \mathfrak{N}$ if and only if $U_{b} \mathfrak{\Im} \subset U_{c} \mathfrak{I}\left(b \in U_{b} \mathfrak{\Re} \subset U_{c} \mathfrak{\Re}\right.$ $\subset U_{c} \mathfrak{I}$ implies $U_{b} \mathfrak{\Im} \subset U_{c} \mathfrak{\Im}$, and conversely $b \in U_{b} \mathfrak{\Im} \subset U_{c} \mathfrak{\jmath}$ implies $b \in \mathfrak{R} \cap U_{c} \mathfrak{\Im}$ $=U_{c} \mathfrak{A}$ so $U_{b} \mathfrak{\Re} \subset U_{c} \mathfrak{\Re}$ ), so chains of principal inner ideals in $\mathfrak{\Re}$ induce corresponding chains in $\mathfrak{\Im}$ so that a chain condition in $\mathfrak{\Im}$ entails one in $\mathfrak{A}$.

COROLlARY. If $\Delta$ is an associative division algebra with involution, $\Delta_{0}$ an ample subspace, then $\mathfrak{\Im}=\mathfrak{S}\left(\Delta_{n}, \Delta_{0}, \gamma\right)$ is regular with a.c.c. and d.c.c. on principal inner ideals.

3. The main theorem. In this section we will classify the inner ideals in the simple algebras $\Im$ of capacity $\geqq 3$. We first establish a lemma which says that, quite generally, inner ideals are "close" to being principal inner ideals $U_{b} \Im$, and in a suitable isotope even close to being an inner ideal $U_{e} \mathfrak{J}$ for $e$ idempotent. For the

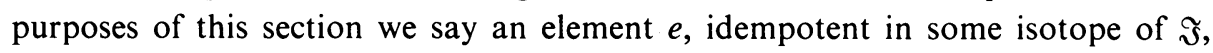
has capacity $m$ if we can write $e=e_{1}+\cdots+e_{m}$ for $e_{i}$ completely primitive idempotents in that isotope.

MAIN LEMMA. Let $\mathfrak{\Im}$ be a regular Jordan algebra with d.c.c. on principal inner ideals. If $\mathfrak{B}$ is an inner ideal in $\mathfrak{\Im}$ there is an isotope $\mathfrak{J}^{(u)}$ and an element $e^{(u)} \in \mathfrak{B}$, idempotent of capacity $m$ in that isotope, such that the Peirce decomposition of $\mathfrak{B}$ in $\mathfrak{\Im}^{(u)}$ relative to $e^{(u)}$ is

$$
\mathfrak{B}=\mathfrak{\Im}_{1}+\mathfrak{B}_{1 / 2} \quad\left(\mathfrak{B}_{1 / 2}=\mathfrak{B} \cap \mathfrak{\Im}_{1 / 2}, \mathfrak{\Im}_{i}=\mathfrak{\Im}_{i}\left(e^{(u)}\right)\right) .
$$

Proof. We want to choose a maximal $e$, but since we are assuming the d.c.c. rather than the a.c.c. we choose a minimal $1-e$ instead. So consider the set of

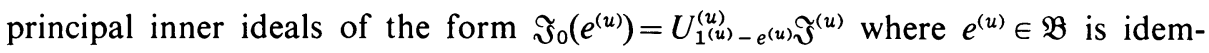
potent with capacity in $\mathfrak{J}^{(u)}$, and choose a minimal one. Since $\mathfrak{B}$ is still inner in $\mathfrak{\Im}^{(u)}$ it suffices to replace $\mathfrak{\Im}$ by $\mathfrak{\Im}^{(u)}$ (and thus avoid cumbersome superscripts). So suppose $e \in \mathfrak{B}$ is an idempotent with capacity such that $\mathfrak{\Im}_{0}=U_{1-e} \mathfrak{\Im}$ contains no $\mathfrak{\Im}_{0}^{(u)}=U_{1^{(u)}}^{(u)}-e^{(u)} \mathfrak{\Im}^{(u)}$ properly.

We have $\mathfrak{B}=\mathfrak{B}_{1}+\mathfrak{B}_{1 / 2}+\mathfrak{B}_{0}\left(\mathfrak{B}_{i}=\mathfrak{B} \cap \mathfrak{\Im}_{i}\right)$ since if $b=b_{1}+b_{1 / 2}+b_{0}$ lies in $\mathfrak{B}$ so do $b_{1}=U_{e} b$ (since $e \in \mathfrak{B}$ ) and $b_{1 / 2}=e \circ b-2 b_{1} \in \mathfrak{B} \circ \mathfrak{B}+\mathfrak{B} \subset \mathfrak{B}$, and therefore $b_{0}=b-b_{1}-b_{1 / 2}$ too. We have $\mathfrak{\Im}_{1}=U_{e} \mathfrak{\Im} \subset \mathfrak{B}$ so $\mathfrak{B}_{1}=\Im_{1}$. We must show $\mathfrak{B}_{0}=0$.

Now $\mathfrak{B}_{0}=\mathfrak{B} \cap \mathfrak{\Im}_{0}$ is an inner ideal in $\mathfrak{\Im}_{0}$, and $\mathfrak{\Im}_{0}$ inherits regularity and the d.c.c. on principal inner ideals from $\Im$, so we saw in Lemma 1 and the Minimal Inner Ideal Theorem that if $\mathfrak{B}_{0}$ is nonzero it contains a minimal inner ideal of the form

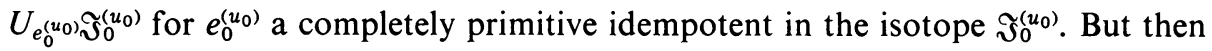
$e^{(u)}=e+e_{0}^{\left(u_{0}\right)} \in \mathfrak{B}$ is an idempotent with capacity in the isotope $\Im^{(u)}$ for $u=e+u_{0}$,

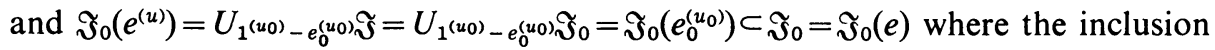


is strict since $e_{0}^{\left(u_{0}\right)} \in \Im_{0}(e)$ but $e_{0}^{\left(u_{0}\right)} \notin \Im_{0}\left(e_{0}^{\left(u_{0}\right)}\right)$. This contradicts the minimality of $\Im_{0}(e)$, so $\mathfrak{B}_{0}$ must be zero.

Let us call a subspace $\mathfrak{B}$ of $\mathfrak{\Im}$ a point space if it consists entirely of points (or rank one elements), elements $b$ which generate a 1-dimensional principal inner ideal $U_{b} \Im=\Phi b$. Note that every subspace of a point space is again a point space, and every point space is an inner ideal.

LEMMA 2. If $\mathfrak{B}_{1 / 2}$ is a subspace of the Peirce space $\mathfrak{\Im}_{1 / 2}$ relative to an idempotent e then $\mathfrak{B}=\mathfrak{I}_{1}+\mathfrak{B}_{1 / 2}$ will be an inner ideal if and only if

$$
\begin{gathered}
U_{\mathfrak{B}_{1 / 2}} \mathfrak{J}_{1}=0, \\
\mathfrak{B}_{1 / 2} \circ \mathfrak{\Im}_{1} \subset \mathfrak{B}_{1 / 2} .
\end{gathered}
$$

In this case any subspace $\mathfrak{B}_{1 / 2}^{\prime} \subset \mathfrak{B}_{1 / 2}$ such that $\mathfrak{B}_{1 / 2}^{\prime} \circ \mathfrak{\Im}_{1} \subset \mathfrak{B}_{1 / 2}^{\prime}$ gives rise to an inner ideal $\mathfrak{B}^{\prime}=\mathfrak{I}_{1}+\mathfrak{B}_{1 / 2}^{\prime} . \mathfrak{B}=\mathfrak{I}_{1}+\mathfrak{B}_{1 / 2}$ will be a point space if and only if

$$
\begin{gathered}
\Im_{1}=\Phi e, \\
U_{\mathfrak{B}_{1 / 2}} \Im_{0}=U_{\mathfrak{B}_{1 / 2}} \Im_{1}=0 .
\end{gathered}
$$

Proof. The conditions are necessary since $U_{\mathfrak{B}_{1 / 2}} \mathfrak{I}_{1} \subset \mathfrak{B} \cap \mathfrak{\Im}_{0}=0$ and $\mathfrak{B}_{1 / 2} \circ \mathfrak{\Im}_{1}$ $=U_{\mathfrak{B}_{1 / 2}, \mathfrak{I}_{1}} e \subset \mathfrak{B} \cap \mathfrak{I}_{1 / 2}=\mathfrak{B}_{1 / 2}$. They are sufficient since they imply (for $b_{1 / 2} \in \mathfrak{B}_{1 / 2}$, $\left.x_{i}, y_{i} \in \Im_{i}\right)$

and hence

$$
\begin{aligned}
U_{b_{1 / 2}, x_{1}} y_{1} & =\left(b_{1 / 2} \circ y_{1}\right) \circ x_{1} \in\left(\mathfrak{B}_{1 / 2} \circ \mathfrak{\Im}_{1}\right) \circ \mathfrak{I}_{1} \subset \mathfrak{B}_{1 / 2}, \\
U_{b_{1 / 2}} x_{1 / 2} & =b_{1 / 2} \circ P_{1}\left\{b_{1 / 2} \circ x_{1 / 2}\right\}-x_{1 / 2} \circ P_{0}\left(b_{1 / 2}^{2}\right) \\
& =b_{1 / 2} \circ P_{1}\left\{b_{1 / 2} \circ x_{1 / 2}\right\} \subset \mathfrak{B}_{1 / 2} \circ \mathfrak{J}_{1} \subset \mathfrak{B}_{1 / 2},
\end{aligned}
$$

$$
\begin{aligned}
U_{\mathfrak{B} \mathfrak{I}}= & U_{\mathfrak{I}_{1}} \mathfrak{\Im}+U_{\mathfrak{B}_{1 / 2}} \mathfrak{\Im}+U_{\mathfrak{B}_{1 / 2}, \mathfrak{I}_{1}} \mathfrak{I} \\
= & \mathfrak{I}_{1}+U_{\mathfrak{B}_{1 / 2}}\left(\mathfrak{I}_{1}+\mathfrak{\Im}_{1 / 2}+\mathfrak{\Im}_{0}\right)+U_{\mathfrak{B}_{1 / 2}, \mathfrak{I}_{1}}\left(\mathfrak{\Im}_{1}+\mathfrak{\Im}_{1 / 2}\right) \\
& \subset \mathfrak{I}_{1}+\left(0+\mathfrak{B}_{1 / 2}+\mathfrak{I}_{1}\right)+\left(\mathfrak{B}_{1 / 2}+\mathfrak{I}_{1}\right)=\mathfrak{B} .
\end{aligned}
$$

If $\mathfrak{B}$ is a point space then $e \in \mathfrak{B}$ has $U_{e} \mathfrak{I}=\Phi e$ so (4) holds; also $U_{\mathfrak{B}_{1 / 2}} \Im_{0}$ $\subset \mathfrak{B}_{1 / 2} \cap \Im_{1}=0$ implies (5). Conversely, if (4), (5) hold and $b=\alpha e+b_{1 / 2} \in \mathfrak{B}$ then $U_{b} e=U_{\alpha e} e+U_{\alpha e, b_{1 / 2}} e=\alpha^{2} e+\alpha\left\{e\right.$ e $\left.b_{1 / 2}\right\}=\alpha b, \quad U_{b} \Im_{0}=U_{b_{1 / 2}} \Im_{0}=0 \quad$ by $\quad(5), \quad U_{b} x_{1 / 2}$ $=\left\{\begin{array}{lll}\alpha e & x_{1 / 2} & b_{1 / 2}\end{array}+U_{b_{1 / 2}} x_{1 / 2}=\alpha P_{1}\left(x_{1 / 2} \circ b_{1 / 2}\right)+b_{1 / 2} \circ P_{1}\left(b_{1 / 2} \circ x_{1 / 2}\right)-x_{1 / 2} \circ P_{0}\left(b_{1 / 2}^{2}\right)\right.$ $=\alpha \beta e+\beta b_{1 / 2}-0=\beta b$ for $P_{1}\left(x_{1 / 2} \circ b_{1 / 2}\right)=\beta e$ by (4). Thus $U_{b} \mathfrak{\Im}=U_{b}\left(\Phi e+\Im_{1 / 2}+\mathfrak{\Im}_{0}\right)$ $\subset \Phi b$ and $b$ is a point.

We can now state our main result.

MAIN THEOREM. Let $\Im$ be a simple unital Jordan algebra of capacity $n \geqq 3$, so $\mathfrak{\Im} \cong \mathfrak{S}\left(\mathfrak{D}_{n}, \mathfrak{D}_{0}, \gamma\right)$ where $\mathfrak{D}$ is either

(i) $\mathfrak{D}=\Delta \oplus \Delta^{0}$ for $\Delta$ an associative division algebra under the exchange involution,

(ii) $\mathfrak{D}=\Delta$ for $\Delta$ an associative division algebra with involution,

(iii) $\mathfrak{D}=\mathfrak{Q}=\Phi_{2}$ a split quaternion algebra with standard involution, 
(iv) $\mathfrak{D}=\mathfrak{C}$ a split Cayley algebra with standard involution $(n=3)$,

(v) $\mathfrak{D}=\mathfrak{C}$ a Cayley division algebra with standard involution $(n=3)$.

In case (ii) or (v) all inner ideals $\mathfrak{B}$ are principal, $\mathfrak{B}=U_{b} \mathfrak{\Im}$, and in some isotope $\mathfrak{B}=U_{e^{(u)}} \mathfrak{\Im}^{(u)}$ for $e^{(u)}$ idempotent in $\mathfrak{\Im}^{(u)}$; the same holds in case (iv) when $\mathfrak{B}$ has

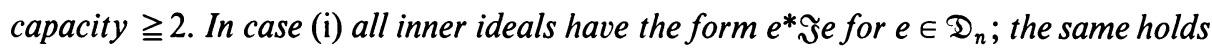
in case (iii) when $\mathfrak{B}$ has capacity $\geqq 2$. In cases (iii) and (iv), every capacity 1 inner ideal is a point space. In case (iii) every maximal point space has the form $\mathfrak{B}=\Phi e_{1}+\mathfrak{Q}_{\varepsilon}[12]$ or $\mathfrak{B}=\Phi e_{1}+\varepsilon \mathfrak{[ 1 2 ]}+\cdots+\varepsilon \mathfrak{[}[1 n]$ in a suitable isotope where $e_{1}, e_{2}, \ldots, e_{n}$ are strongly connected completely primitive idempotents in $\Im^{(u)}$ and $\varepsilon$ a primitive idempotent in $\mathfrak{Q}$. In case (iv), every maximal point space has either the form $\mathfrak{B}=\Phi e_{1}+\mathfrak{V}_{\varepsilon}[12]$ or the form $\mathfrak{B}=\Phi e_{1}+\varepsilon \mathfrak{E}[12]+\Phi_{\varepsilon}[13]$ in a suitable isotope for $e_{1}, e_{2}, e_{3}$ strongly connected idempotents in $\Im^{(u)}$ and $\varepsilon$ a primitive idempotent in $\mathfrak{E}$.

Proof. By the Main Lemma, $\mathfrak{B}=\mathfrak{I}_{1}+\mathfrak{B}_{1 / 2}$ relative to $e=e_{1}+\cdots+e_{m}$ in some isotope, where $e_{1}, \ldots, e_{n}$ are strongly connected primitive idempotents in $\mathfrak{\Im}^{(u)}$. Relative to this family we obtain an isomorphism $\mathfrak{\Im}^{(u)}=\mathfrak{S}\left(\mathfrak{D}_{n}, \mathfrak{D}_{0}\right)$ by the Coordinatization Theorem [2], [3] in such a way that the $e_{i}$ correspond to the diagonal idempotents $1[i i]$. Since $\mathfrak{J}$ is simple, by the Herstein-Kleinfeld-Osborn Theorem we have the possibilities (i)-(v) for $\mathfrak{D}$. Replacing $\Im$ by $\mathfrak{\Im}^{(u)}$, assume $\mathfrak{F}=\mathfrak{S}\left(\mathfrak{D}_{n}, \mathfrak{D}_{0}\right)$.

We use this coordinatization to draw further information from the decomposition $\mathfrak{B}=\mathfrak{I}_{1}+\mathfrak{B}_{1 / 2}$. Write $b \in \mathfrak{B}_{1 / 2}$ as $b=\sum x_{i j}(b)[i j]$ for $x_{i j}(b) \in \mathfrak{D}, 1 \leqq i \leqq m$, $m+1 \leqq j \leqq n$. (We use $i, i^{\prime}$ for indices between 1 and $m$, corresponding to the idempotents $e_{k k} \in \mathfrak{B}$, and $j, j^{\prime}$ for indices between $m+1$ and $n$, corresponding to $e_{k k} \notin \mathfrak{B}$.) The condition (3) that $\mathfrak{I}_{1} \circ \mathfrak{B}_{1 / 2} \subset \mathfrak{B}_{1 / 2}$ amounts to $d\left[i^{\prime} i\right] \circ b \in \mathfrak{B}_{1 / 2}$, $d_{0}[i i] \circ b \in \mathfrak{B}_{1 / 2}$ for $d \in \mathfrak{D}, d_{0} \in \mathfrak{D}_{0}$ since $\mathfrak{\Im}_{1}=\sum \mathfrak{D}_{0}[i i]+\sum \mathfrak{D}\left[i^{\prime} i\right]$, hence

$$
\begin{gathered}
\mathfrak{B}_{1 / 2}=\mathfrak{B}_{1 / 2,1} \oplus \cdots \oplus \mathfrak{B}_{1 / 2, m} \quad\left(\mathfrak{B}_{1 / 2, i}=\mathfrak{B} \cap \mathfrak{I}_{1 / 2}\left(e_{i}\right)=e_{i} \circ \mathfrak{B}_{1 / 2}\right), \\
x_{i j}\left(d_{0}[i i] \circ b\right)=d_{0} x_{i j}(b) \quad\left(d_{0} \in \mathfrak{D}_{0}\right), \\
x_{i^{\prime} j}\left(d\left[i^{\prime} i\right] \circ b\right)=d x_{i j}(b) \quad(d \in \mathfrak{D}) .
\end{gathered}
$$

The condition (2) that $U_{\mathfrak{B}_{1 / 2}} \mathfrak{\Im}_{1}=0$ amounts to

and

$$
U_{x_{i j}(b)[i j]} d_{0}[i i]=\left\{x_{i j}(b)[i j] d_{0}[i i] x_{i j^{\prime}}(b)\left[i j^{\prime}\right]\right\}=0
$$

hence

$$
\begin{aligned}
\left\{x_{i j}(b)[i j] d\left[i i^{\prime}\right] x_{i^{\prime} j}(b)\left[i^{\prime} j\right]\right\}= & \left\{x_{i j}(b)[i j] d\left[i i^{\prime}\right] x_{i^{\prime} j^{\prime}}(b)\left[i^{\prime} j^{\prime}\right]\right\} \\
& +\left\{x_{i j^{\prime}}(b)\left[i j^{\prime}\right] d\left[i i^{\prime}\right] x_{i^{\prime} j}(b)\left[i^{\prime} j\right]\right\}=0,
\end{aligned}
$$

$$
\begin{aligned}
& \overline{x_{i j}(b)} \mathfrak{D}_{0} x_{i j}(b)=0, \\
& \overline{x_{i j}(b)} \mathfrak{D}_{0} x_{i j^{\prime}}(b)=0,
\end{aligned}
$$

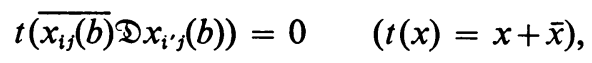

$$
\begin{aligned}
& \overline{x_{i j}(b)} d x_{i^{\prime} j^{\prime}}(b)+\overline{\overline{x_{i j^{\prime}}(b)} d x_{i^{\prime} j}(b)}=0 \text {. }
\end{aligned}
$$


Let $\mathfrak{F}_{i j}$ denote the subspace of $\mathfrak{D}$ of elements of the form $x_{i j}(b)$ for $b \in \mathfrak{B}_{1 / 2}$. By (9), if the norm of $x \in \mathfrak{D}$ is $n(x)=\bar{x} x$, then

$$
n\left(\mathfrak{ほ}_{i j}\right)=0 .
$$

Also, if the capacity is $m \geqq 2$ then there exist distinct $i, i^{\prime}$ and (8) shows $\mathscr{D} \mathscr{E}_{i j} \subset \mathfrak{E}_{i^{\prime} j}$. In particular, $\mathfrak{E}_{i j} \subset \mathfrak{E}_{i^{\prime} j}$ for all $i \neq i^{\prime}$, so all $\mathfrak{E}_{i j}$ have a common value $\mathfrak{E}_{j}$ which is a left ideal:

$$
\mathfrak{E}_{i j}=\mathfrak{E}_{j}(1 \leqq i \leqq m) \quad \text { where } \mathfrak{D E}_{j} \subset \mathfrak{E}_{j}(\text { if } m \geqq 2) .
$$

Right away we can get rid of cases (ii) and (v) and case (iv) when $m \geqq 2$; we claim $\mathfrak{B}_{1 / 2}=0, \mathfrak{B}=\mathfrak{\Im}_{1}=U_{e} \mathfrak{\Im}$ is principal. Indeed, since $\mathfrak{D}$ is a division algebra in cases (ii), (v) the formula (13) shows $\mathfrak{F}_{i j}=0, x_{i j}(b)=0$, and $b=\sum x_{i j}(b)[i j]=0$ for $b \in \mathfrak{B}_{1 / 2}$. A similar argument works for case (iv) when $m \geqq 2$; here $\mathfrak{D}$ is not a division algebra, but it still contains no proper left ideals, so by $(14) \mathbb{E}_{i j}$ is an ideal which cannot be all of $\mathfrak{D}$ by $(13)$, and $\mathfrak{E}_{i j}=0$.

Next, let us turn to case (i). Write $x_{i j}(b) \in \mathfrak{D}=\Delta \oplus \Delta^{0}$ as $y_{i j}(b) \oplus z_{i j}(b)$. Then (9) and (10) imply $z_{i j}(b) y_{i k}(b)=0$ for all $j, k$, and since $\Delta$ is a division algebra for any $b$ we have either $z_{i j}(b)=0$ or $y_{i k}(b)=0$. But then a standard argument shows either $z_{i j}(b)=0$ for all $b$ or $y_{i k}(b)=0$ for all $b\left(j, k\right.$ fixed), therefore $z_{i j}(\mathfrak{B})=0$ for all $j$ or $y_{i j}(\mathfrak{B})=0$ for all $j$. Furthermore, all indices $i$ behave in the same way: if $y_{i j}(\mathfrak{B}) \neq 0$ but all $z_{i j}(\mathfrak{B})=0$ then for any $i^{\prime}$ we have $z_{i^{\prime} j}(\mathfrak{B})=0$ for all $j$ because of (14). Thus we may assume (say) $z_{i j}(\mathfrak{B})=0$ for all $i, j$ (dually if all $y_{i j}(\mathfrak{B})=0$ ). This means $b=\sum x_{i j}(b)[i j]=\sum\left\{y_{i j}(b) \oplus 0\right\}[i j]=\sum y_{i j}(b) \varepsilon[i j]$ for $\varepsilon=1 \oplus 0 \in \Delta \oplus \Delta^{0}=\mathfrak{D}$. Suppose $y_{1 m+1}, \ldots, y_{1 m+r}$ form a basis for the space of linear functionals $\mathfrak{B} \rightarrow \Delta$ spanned by $y_{1 m+1}, \ldots, y_{1 n}: y_{1 m+s}=\sum y_{1 j} \delta_{j s}$ for $s=r+1, \ldots, n-m$. By (8) this implies $y_{i m+s}=\sum y_{i j} \delta_{j s}$ for all $i$. Thus the component of $b$ in $\mathfrak{B}_{1 / 2, i}$ (as in (6)) is $y_{i}(b)$ $=\sum_{m+1}^{m+r} y_{i j}(b) \varepsilon[i j]+\sum_{s} y_{i j}(b) \delta_{j s} \varepsilon[i m+s]=\sum_{m+1}^{m+r} y_{i j}(b) \varepsilon[i j]^{\prime} \quad$ where $d[i j]^{\prime}=d[i j]$ $+\sum d \delta_{j s}[i m+s]=d e_{i j} e_{j j}^{\prime}+\bar{d} e_{j j}^{\prime} e_{j i}$ for $e_{j j}^{\prime}=e_{j j}+\sum \delta_{j s}[j m+s]$ are orthogonal idempotents in $\mathfrak{J}=\mathfrak{S}\left(\Delta_{n} \oplus \Delta_{n}^{0}\right)$ strongly connected to $e_{i i}$ by $1[i j]^{\prime}$. Using the $e_{j j}^{\prime}$ in place of the $e_{j j}$ we obtain a coordinatization of $\mathfrak{I}$ relative to which $\mathfrak{B}_{1 / 2}=\oplus \mathfrak{B}_{1 / 2, i}$ $=\oplus \mathscr{D} \varepsilon[i j]^{\prime}$ and $\mathfrak{B}=e^{*} \mathfrak{\jmath} e$ for $e=\sum e_{i i}+\varepsilon \sum e_{j j}^{\prime}$.

Before turning to case (iii) in capacity $m \geqq 2$ we find it convenient to prove two lemmas.

LEMMA 3. If relative to $e_{1}, \ldots, e_{n}$ the element $b=x_{m+1}[1 m+1]+\cdots+x_{n}[1 n]$ $\in \mathfrak{F}_{\mathfrak{C}}\left(\mathfrak{D}_{n}\right)(\mathfrak{D}=\mathfrak{Q}$ or $\mathfrak{C})$ has $U_{b} e_{1}=0$ then there are primitive orthogonal idempotents $e_{1}^{\prime}, \ldots, e_{n}^{\prime}$ with $e_{1}^{\prime}=e_{1}, \ldots, e_{m}^{\prime}=e_{m}$ such that $b \in \mathfrak{J}_{1 n}^{\prime}=U_{e_{1}^{\prime}, e_{n}^{\prime}} \mathfrak{.}$.

Proof. For convenience we assume $m=1$; choose $e_{2}, \ldots, e_{n}$ so that $b$ has as few nonzero entries in $\mathfrak{\Im}_{12}, \ldots, \mathfrak{\Im}_{1 n}$ as possible. We claim there is just one such entry. Otherwise there are two-for convenience say $b=\sum x_{i}[1 i]$ where $x_{2}, x_{3} \neq 0$. The components in $\Im_{22}$ and $\Im_{23}$ of $U_{b} e_{1}$ are $U_{x_{2}[12]} e_{1}=n\left(x_{2}\right) e_{2}$ and $\left\{x_{3}[13] e_{1} x_{2}[12]\right\}$ $=\bar{x}_{3} x_{2}$ [32], so $n\left(x_{2}\right)=0$ and $\bar{x}_{3} x_{2}=0$. By nondegeneracy of the norm form on 
$\mathscr{D}=\mathfrak{Q}$ or $\mathbb{C}$ there is $x \in \mathscr{D}$ with $n\left(x, x_{3}\right)=1$; then $x_{2}=n\left(x, x_{3}\right) x_{2}=x\left(\bar{x}_{3} x_{2}\right)+x_{3}\left(\bar{x} x_{2}\right)$ $=x_{3} a$ where $a=\bar{x} x_{2}$ has $n(a)=n(\bar{x}) n\left(x_{2}\right)=0$.

Consider $e_{2}^{\prime}=e_{2}-a$ [32], $e_{3}^{\prime}=e_{3}+a$ [32], $e_{i}^{\prime}=e_{i}$ for $i \neq 2,3$. Since $n(a)=0, e_{2}^{\prime}$ and $e_{3}^{\prime}$ are primitive orthogonal idempotents, $e_{2}^{\prime}+e_{3}^{\prime}=e_{2}+e_{3}$, so the $e_{i}^{\prime}$ form a primitive orthogonal family. The components of $b$ in $\mathfrak{\Im}_{1 i}^{\prime}$ for $i=4, \ldots, n$ are the same as those in $\mathfrak{J}_{1 i}$ since $\left\{e_{1} b e_{i}^{\prime}\right\}=\left\{e_{1} b e_{i}\right\}$, but the component in $\mathfrak{\Im}_{12}$ is now zero since

$$
\begin{aligned}
\left\{e_{1} b e_{2}^{\prime}\right\} & =\left\{e_{1} b e_{2}\right\}-\left\{e_{1} b a[32]\right\} \\
& =x_{2}[12]-\left\{e_{1} x_{2}[12] \bar{a}[23]\right\}-\left\{e_{1} x_{3}[13] a[32]\right\} \\
& =\left\{x_{2}-x_{3} a\right\}[12]-x_{2} \bar{a}[13]=0
\end{aligned}
$$

where $x_{2}=x_{3} a$ and $x_{2} \bar{a}=\left(x_{3} a\right) \bar{a}=x_{3} n(a)=0$. Thus we have one less component relative to $e_{2}^{\prime}, \ldots, e_{n}^{\prime}$, contradicting the minimality of $e_{2}, \ldots, e_{n}$. This means there could only have been one nonzero component to begin with.

Lemma 4. Any totally isotropic subspace $\mathfrak{E}_{0}$ of the split Cayley algebra $\mathfrak{E}$ is isotopic to one of

(i) $\Phi \varepsilon_{11}$,

(ii) $\Phi_{\varepsilon_{11}}+\Phi_{\varepsilon_{10}}$ or $\Phi_{\varepsilon_{11}}+\Phi_{\varepsilon_{01}}$,

(iii) $\Phi_{\varepsilon_{11}}+\Phi_{\varepsilon_{10}}+\Phi_{\eta_{10}}$ or $\Phi_{\varepsilon_{11}}+\Phi_{\varepsilon_{01}}+\Phi \eta_{01}$

(iv) $\varepsilon_{11} \mathfrak{E}=\Phi \varepsilon_{11}+\Phi \varepsilon_{10}+\Phi \eta_{10}+\Phi \nu_{10}$ or $\mathfrak{\mho}_{\varepsilon_{11}}=\Phi_{\varepsilon_{11}}+\Phi \varepsilon_{01}+\Phi \eta_{01}+\Phi \nu_{01}$

according to its dimension, where $\varepsilon_{11}, \varepsilon_{00}, \varepsilon_{10}, \varepsilon_{01}, \eta_{10}, \eta_{01}, \nu_{10}, \nu_{01}$ is a basis of Cayley matrix units for $\mathfrak{c}$.

There is an isomorphism $\mathfrak{S}_{(}\left(\mathfrak{S}_{3}, \gamma\right) \rightarrow \mathfrak{S}_{(}\left(\mathfrak{E}_{3}, \gamma^{\prime}\right)$ sending $e_{i} \rightarrow e_{i}$ and $\mathfrak{E}_{0}[12] \rightarrow \mathfrak{E}_{0}^{\prime}[12]$ where $\mathfrak{C}_{0}^{\prime}$ is one of (i)-(iv). Similarly, in the case of a totally isotropic subspace $\mathfrak{Q}_{0}$ of a split quaternion algebra $\mathfrak{Q}$ there is an isomorphism $\mathfrak{\mathfrak { S }}\left(\mathfrak{Q}_{n}, \gamma\right) \rightarrow \mathfrak{5}\left(\mathfrak{Q}_{n}, \gamma^{\prime}\right)$ sending $e_{i} \rightarrow e_{i}$ and $\mathfrak{Q}_{0}[1 n] \rightarrow \mathfrak{Q}_{0}^{\prime}[1 n]$ where $\mathfrak{Q}_{0}^{\prime}$ is one of (i)-(ii).

Proof. By Witt's Theorem, every totally isotropic subspace $\mathfrak{E}_{0}$ of $\mathfrak{C}$ is conjugate to one of the first members of the pairs (i)-(iv) under an orthogonal transformation, and conjugate to either the first or second member of the pair under a proper orthogonal transformation $t$. Then [8, p. 161] we have $t(x y)=t_{1}(x) t_{2}(y)$ for proper orthogonal $t_{1}, t_{2}$. This implies $t_{1}(x)=t(x) u, t_{2}(x)=v t(y)$, and $t(x y)=\{t(x) u\}\{v t(y)\}$ for $u=t_{2}(1)^{-1}, v=t_{1}(1)^{-1}$. Thus $t$ is an isomorphism of $\mathbb{E}$ with its $u, v$-isotope $\mathfrak{\mho}^{(u, v)}$ (see [7]) sending $\mathfrak{E}_{0}$ to one of the above standard models $\mathbb{\mathfrak { S }}_{0}^{\prime}$.

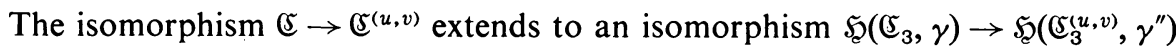
sending $e_{i} \rightarrow e_{i}$ and $\mathfrak{E}_{0}[i j] \rightarrow \mathfrak{E}_{0}^{\prime}[i j]$. On the other hand, the elements $(u v)^{-1}[12]$,

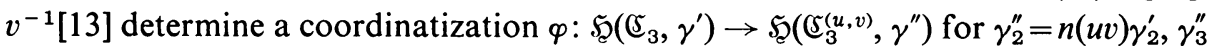
$=n(v) \gamma_{3}^{\prime}$ by [7, Theorem 3]. Turning this around, for $\gamma_{2}^{\prime}=n(u v)^{-1} \gamma_{2}^{\prime \prime}, \gamma_{3}^{\prime}=n(v)^{-1} \gamma_{3}^{\prime \prime}$ we have $\varphi^{-1}: \mathfrak{S}\left(\mathfrak{C}_{3}^{(u, v)}, \gamma^{\prime \prime}\right) \rightarrow \mathfrak{S}\left(\mathfrak{E}_{3}, \gamma^{\prime}\right)$ sending $e_{i} \rightarrow e_{i}, \mathfrak{C}_{0}^{\prime}[12] \rightarrow \mathfrak{C}_{0}^{\prime}[12]$ ( $\varphi$ corresponds to the identity map on $\mathbb{E}[12] \rightarrow \mathfrak{C}^{(u, v)}[12]$, though not on the other $\left.\mathfrak{E}[i j]\right)$. Composing these, we obtain the desired $\mathfrak{S}\left(\mathfrak{E}_{3}, \gamma\right) \rightarrow \mathfrak{S}\left(\mathfrak{E}_{3}, \gamma^{\prime}\right)$.

A similar process works in the quaternion case. 
To return to case (iii), $m \geqq 2$, if $\mathfrak{B}_{1 / 2} \neq 0$ then from (6) $\mathfrak{B}_{1 / 2}=\oplus \mathfrak{B}_{1,2, i}$ we can assume (for convenience) $\mathfrak{B}_{1 / 2,1} \neq 0$ : some $b_{0}=\sum x_{1 j}\left(b_{0}\right)[1 j] \neq 0$. Applying the lemmas we can assume $b_{0}=\varepsilon[1 n]$. By linearizing (12) with $i^{\prime}=1, j^{\prime}=n$ we have

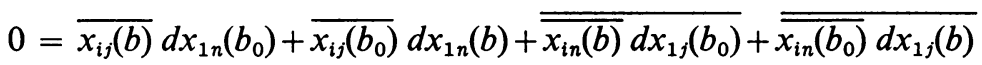

$$
\begin{aligned}
& =\overline{x_{i j}(b)} d \varepsilon \quad \text { for all } b \in \mathfrak{B}, d \in \mathfrak{D} \text {. }
\end{aligned}
$$

By primeness of $\mathfrak{D}=\mathfrak{Q}$ we get $x_{i j}(b)=0$ for all $b$ as long as $i \neq 1, j \neq n$. But then $\mathfrak{E}_{i j}=0$ for $i \neq 1, j \neq n$ implies $\mathfrak{F}_{j}=0$ for $j \neq n$ by (14), while $\varepsilon \in \mathfrak{E}_{n}$ implies $\mathfrak{E}_{n} \supset \mathfrak{D}_{\varepsilon}$; since $\mathfrak{D} \varepsilon$ is maximal isotropic, by $(13)$ we have $\mathfrak{E}_{n}=\mathscr{D} \varepsilon$. Thus $\mathfrak{B}_{1 / 2, i}=\mathfrak{E}_{i n}[$ in $]=\mathfrak{E}_{n}[$ in $]$ $=\mathfrak{D}_{\varepsilon}[$ in $]$ and $\mathfrak{B}_{1 / 2}=\oplus \mathfrak{B}_{1 / 2, i}=\mathfrak{D}_{\varepsilon}[1 n]+\cdots+\mathfrak{D}_{\varepsilon}[m n]$. It is easily verified that $\mathfrak{B}=e^{*} \mathfrak{D}_{n} e \cap \mathfrak{\Im}=e^{*} \mathfrak{\Im} e$ for $e=e_{11}+\cdots+e_{m m}+\varepsilon e_{n n}$ in $\mathfrak{D}_{n}$ (note $\left(\bar{\varepsilon} e_{n n}\right) \mathfrak{D}_{0}[n n]\left(\varepsilon e_{n n}\right)$ $\left.=\Phi_{\bar{\varepsilon} \varepsilon} e_{n n}=0\right)$.

Finally, we must consider the case of capacity $m=1$ in (iii) and (iv). We begin with (iii), $\mathfrak{B}=\Phi e_{1}+\mathfrak{B}_{1 / 2}$ where any $b \in \mathfrak{B}_{1 / 2}$ can be written $b=x_{2}(b)[12]+\cdots$ $+x_{n}(b)[1 n]$. By the lemmas we may assume $b_{0}=\varepsilon[12] \in \mathfrak{B}_{1 / 2}$. From (9), (10)

$$
\overline{x_{j}(b)} x_{k}(b)=0
$$

so linearization gives

$$
\overline{x_{j}(b)} x_{k}\left(b_{0}\right)+\overline{x_{j}\left(b_{0}\right)} x_{k}(b)=0 .
$$

For $j=2, k \neq 2$ we have $x_{2}\left(b_{0}\right)=\varepsilon, x_{k}\left(b_{0}\right)=0$ so $\bar{\varepsilon} x_{k}(b)=0$. In $\mathfrak{D}$ we have $\varepsilon=\varepsilon_{11}$, $\bar{\varepsilon}=\varepsilon_{00}=1-\varepsilon_{11}$ so this says $x_{k}(b) \in \varepsilon \mathfrak{D}=\mathfrak{D}_{11}+\mathfrak{D}_{10}=\Phi \varepsilon_{11}+\Phi \varepsilon_{10} \quad\left(\varepsilon_{i j}\right.$ the matrix units for $\mathscr{D}=\Phi_{2}$ ):

$$
x_{k}(b)=\alpha_{k 11}(b) \varepsilon_{11}+\alpha_{k 10}(b) \varepsilon_{10} \quad(k \neq 2) .
$$

For $k=2$ we get

$$
\begin{gathered}
\overline{x_{2}(b)} \varepsilon+\bar{\varepsilon} x_{2}(b)=0, \quad \bar{\varepsilon} x_{2}(b) \bar{\varepsilon}=0, \\
x_{2}(b)=\alpha_{211}(b) \varepsilon_{11}+\alpha_{210}(b) \varepsilon_{10}+\alpha_{201}(b) \varepsilon_{01} .
\end{gathered}
$$

Then

$$
\begin{aligned}
0=\overline{x_{k}(b)} x_{2}(b) & =\left\{\alpha_{k 11}(b) \varepsilon_{00}-\alpha_{k 10}(b) \varepsilon_{10}\right\}\left\{\alpha_{211}(b) \varepsilon_{11}+\alpha_{210}(b) \varepsilon_{10}+\alpha_{201}(b) \varepsilon_{01}\right\} \\
& =\alpha_{k 11}(b) \alpha_{201}(b) \varepsilon_{01}-\alpha_{k 10}(b) \alpha_{201}(b) \varepsilon_{11}
\end{aligned}
$$

and

$$
\begin{aligned}
0 & =\overline{x_{2}(b)} x_{2}(b) \\
& =\left\{\alpha_{211}(b) \varepsilon_{00}-\alpha_{210}(b) \varepsilon_{10}-\alpha_{201}(b) \varepsilon_{01}\right\}\left\{\alpha_{211}(b) \varepsilon_{11}+\alpha_{210}(b) \varepsilon_{10}+\alpha_{201}(b) \varepsilon_{01}\right\} \\
& =-\alpha_{210}(b) \alpha_{201}(b) \varepsilon_{11}-\alpha_{201}(b) \alpha_{210}(b) \varepsilon_{00}
\end{aligned}
$$

imply

$$
\alpha_{k 11}(b) \alpha_{201}(b)=\alpha_{k 10}(b) \alpha_{201}(b)=\alpha_{210}(b) \alpha_{201}(b)=0
$$


There are two possibilities: if $\alpha_{201}(b)$ vanishes identically then $x_{k}(b) \in \varepsilon \mathfrak{D}$, $x_{2}(b) \in \varepsilon \mathfrak{D}$ so $\mathfrak{B}_{1 / 2} \subset \varepsilon \mathfrak{D}[12]+\cdots+\varepsilon \mathfrak{D}[1 n]$ where $\mathfrak{B}^{\prime}=\Phi e_{1}+\varepsilon \mathfrak{D}[12]+\cdots+\varepsilon \mathfrak{T}[1 n]$ is already a maximal point space. On the other hand, if the function $\alpha_{201}$ does not vanish identically then by (15) and its linearization $\alpha_{k 11}=\alpha_{k 10}=\alpha_{210}=0, x_{k}(b)=0$ for $k \neq 2$ and $x_{2}(b) \in \mathfrak{D}_{11}+\mathfrak{D}_{01}=\mathfrak{D}_{\varepsilon}, \mathfrak{B}_{1 / 2} \subset \mathfrak{D} \varepsilon[12]$ where again $\mathfrak{B}^{\prime \prime}=\Phi e_{1}+\mathfrak{D} \varepsilon[12]$ is a maximal point space.

A more complicated argument applies to case (iv) when $m=1$. Following the approach of T. A. Springer and F. Veldkamp [9], we show that every point space $\mathfrak{B}$ of dimension $\leqq 5$ can be imbedded in a Peirce space $\mathfrak{\Im}_{0}\left(e_{3}\right)$ for some $e_{3}$ in some isotope.

We will have occasion to use the following criterion: if we can find $\alpha, \beta \in \Phi$ and $a \in \mathbb{C}$ such that

(i) $\alpha \beta=n(a)$,

$$
\begin{aligned}
& \text { (ii) for all } b=\lambda e_{1}+x_{2}[12]+x_{3}[13] \text { in } \mathfrak{B}=\Phi e_{1}+\mathfrak{B}_{1 / 2}, \alpha x_{2}+x_{3} \bar{a}=0 \text {, } \\
& \beta x_{3}+x_{2} a=0
\end{aligned}
$$

then in $\tilde{\Im}=\Im^{\left(u^{-1}\right)}, u=e_{1}+c[23]$ where

(iii) $n(a, c)=1, n(c) \neq 0$

(such elements always exist for $a \neq 0$ ), the element $\tilde{e}_{3}=U_{u} w$ for $w=\alpha e_{2}+\beta e_{3}+a[23]$ is a primitive idempotent with $\mathfrak{B} \subset \tilde{\Im}_{0}\left(\tilde{e}_{3}\right)$. To see this, note $\tilde{U}_{\tilde{e}_{3}}=U_{\tilde{e}_{3}} U_{u}-1=U_{u} U_{w}$ and $\tilde{V}_{\tilde{e}_{3}}=V_{\tilde{e}_{3}, u^{-1}}=V_{u, w}$. The condition that $\tilde{e}_{3}$ be idempotent in $\tilde{\Im}$ is $w=U_{w} u$ $=\{\alpha \beta c+a \bar{c} a\}[23]+\alpha n(a, c) e_{2}+\beta n(a, c) e_{3}$, so the result follows from (iii) (observe $\alpha \beta c+a \bar{c} a=n(a) c+\{n(a, c) a-c n(a)\}=a)$. The condition that $\tilde{e}_{3}$ be primitive is that $w$ be rank 1 , which follows from (i). The conditions $\tilde{U}_{\tilde{e}_{3}} \mathfrak{B}=\tilde{V}_{\tilde{e}_{3}} \mathfrak{B}=0$ that $\mathfrak{B}$ be contained in $\tilde{\Im}_{0}\left(\tilde{e}_{3}\right)$ reduce to $U_{w} \mathfrak{B}=\{u w \mathfrak{B}\}=0$; the first is automatic since $w \in \Im_{0}\left(e_{1}\right)$, as is $\left\{u w \Phi e_{1}\right\}=0$, while $\left\{u w x_{2}[12]+x_{3}[13]\right\}=\left\{\beta x_{3}+x_{2} a\right\} \bar{c}[12]$ $+\left\{\alpha x_{2}+x_{3} \bar{a}\right\} c[13]=0$ by (ii).

If $b=x_{2}[12]+x_{3}[13] \in \mathfrak{B}$ where $b_{1}=\varepsilon[12] \in \mathfrak{B}$ then the conditions

$$
\overline{x_{2}(b)} x_{3}\left(b_{1}\right)+\overline{x_{2}\left(b_{1}\right)} x_{3}(b)=0
$$

imply $\bar{\varepsilon} x_{3}=0$,

$$
x_{3}(b)=\alpha_{311}(b) \varepsilon_{11}+x_{310}(b) \quad\left(x_{310}(b) \in \mathfrak{C}_{10}\right) .
$$

Similarly

$$
\overline{x_{2}(b)} x_{2}\left(b_{1}\right)+\overline{x_{2}\left(b_{1}\right)} x_{2}(b)=0
$$

implies $\bar{\varepsilon} x_{2}(b) \bar{\varepsilon}=0$, and by subtracting a suitable multiple of $b_{1}$ we can also arrange that $\varepsilon x_{2}(b) \varepsilon=0$ :

$$
x_{2}(b)=x_{210}(b)+x_{201}(b) \quad\left(x_{2 i j}(b) \in \mathfrak{C}_{i j}\right)
$$


The condition $n\left(x_{2}(b)\right)=0$ and $\overline{x_{2}(b)} x_{3}(b)=0$ leads to

(19) $x_{201}(b) \perp x_{210}(b)$,

(20) $x_{201}(b) \perp x_{310}(b)$,

(21) $\alpha_{311}(b) x_{201}(b)=x_{310}(b) x_{210}(b)$

(where $x \perp y$ means $t(x, y)=0$ ). If $b_{2}=\varepsilon_{10}[12] \in \mathfrak{B}$ then similarly $\varepsilon_{10} x_{3}=0$, and $\varepsilon_{01} x_{3}=0$ if $\varepsilon_{01}[12] \in \mathfrak{B}$ :

$$
\begin{gathered}
\varepsilon_{10} x_{310}(b)=0 \quad \text { if } \varepsilon_{10}[12] \in \mathfrak{B}, \\
\alpha_{311}(b)=0, \varepsilon_{01} \perp x_{310}(b) \quad \text { if } \varepsilon_{01}[12] \in \mathfrak{B},
\end{gathered}
$$

while from $n\left(x_{2}\right)=0$ we get by linearization

$$
\begin{aligned}
& \varepsilon_{10} \perp x_{201}(b) \quad \text { if } \varepsilon_{10}[12] \in \mathfrak{B}, \\
& \varepsilon_{01} \perp x_{210}(b) \text { if } \varepsilon_{01}[12] \in \mathfrak{B} .
\end{aligned}
$$

The case $\operatorname{dim} \mathfrak{B}=1$ is an immediate application of the Main Lemma:

$$
\mathfrak{B}_{1}=\Phi e_{1}
$$

Similarly, if $\operatorname{dim} \mathfrak{B}=2$ by Lemmas 3,4 we may take

$$
\mathfrak{B}_{2}=\Phi e_{1}+\Phi \varepsilon_{11}[12]
$$

Consider the case $\operatorname{dim} \mathfrak{B}=3$; we may assume $\mathfrak{B}_{3}=\mathfrak{B}_{2}+\Phi b_{2}$ for $\mathfrak{B}_{2}$ as in (27), $b_{2}=x_{2}[12]+x_{3}[13]$. If we take $\alpha=\beta=0, a=x_{201}$ (as in (18)) then condition (16) is met (using (19), (20)) as long as $x_{201} \neq 0$, so by passing to an isotope we may assume $\mathfrak{B}_{3}=\Phi e_{1}+\mathfrak{C}_{0}[12]$ for $\mathfrak{C}_{0}$ a 2-dimensional totally isotropic subspace of $\mathfrak{E}$. If $x_{201}=0$ we take $\alpha=\beta=0, a=a_{01}$ in $\mathfrak{夭}_{01}$ with $a_{01} \perp x_{310}, x_{210}$ (which is always possible). By Lemma 4 we may arrange it so that

$$
\mathfrak{B}_{3}=\Phi e_{1}+\left(\Phi \varepsilon_{11}+\Phi \varepsilon_{10}\right)[12] \text { or } \mathfrak{B}_{3}=\Phi e_{1}+\left(\Phi \varepsilon_{11}+\Phi \varepsilon_{01}\right)[12] \text {. }
$$

Write $\mathfrak{B}_{4}=\mathfrak{B}_{3}+\Phi b_{3}$ for $\mathfrak{B}_{3}$ as in (28), $b_{3}=x_{2}[12]+x_{3}$ [13]. In the first possibility of (28) we take $\alpha=\beta=0, a=x_{201}$ and again obtain (16) using (24) for $b_{2}$ and as before for $b_{1}, b_{3}$ (as long as $x_{201} \neq 0$; when $x_{201}=0$ we take $\alpha=\beta=0, a=a_{01} \in \mathfrak{C}_{01}$ where $a_{01} \perp \varepsilon_{10}, x_{310}, x_{210}$, which is possible since (22) implies $x_{310} \in \Phi \varepsilon_{10}$ because $y_{10} z_{10}=0$ implies $y_{10}, z_{10}$ in $\mathfrak{S}_{10}$ are linearly dependent). Similarly, in the second possibility of (28) we may assume $x_{3} \neq 0$ (or else $\mathfrak{B}_{4} \subset \Phi e_{1}+\subseteq[12]$ already), and $\alpha_{311}=0$ by (23), so $x_{310} \neq 0$. Then (21) $x_{310} x_{210}=0$ implies $x_{210}=-\beta x_{310}$ for some $\beta$, and if we take this $\beta$ together with $\alpha=0, a=\varepsilon_{00}$ we obtain (16) by (23), (25), and the definition of $\beta$. Thus in all cases we get $\mathfrak{B}_{4} \subset \Phi e_{1}+\subseteq[12]$, and by Lemma 4 again we can assume

$$
\begin{aligned}
& \mathfrak{B}_{4}=\Phi e_{1}+\left(\Phi \varepsilon_{11}+\Phi \varepsilon_{10}+\Phi \eta_{10}\right)[12] \text { or } \\
& \mathfrak{B}_{4}=\Phi e_{1}+\left(\Phi \varepsilon_{11}+\Phi \varepsilon_{01}+\Phi \eta_{01}\right)[12]
\end{aligned}
$$


Write $\mathfrak{B}_{5}=\mathfrak{B}_{4}+\Phi b_{4}, b_{4}=x_{2}[12]+x_{3}[13]$ as always. Consider the first case in (29). Linearizing (21) gives $x_{310} \varepsilon_{10}=x_{310} \eta_{10}=0 \quad\left(x_{i j k}=x_{i j k}\left(b_{4}\right)\right)$, so $x_{310}=0$. If $\alpha_{311}=0$ then $x_{3}=0$ and already $\mathfrak{B}_{4} \subset \Phi e_{1}+\mathfrak{C}[12]$. Otherwise (21) yields $\alpha_{311} x_{201}=0$ so $x_{201}=0, b_{4}=x_{210}[12]+\alpha_{311} \varepsilon_{11}[13]$, so (by subtracting off suitable multiples of $\left.\varepsilon_{10}[12], \eta_{10}[12]\right)$ we can assume $b_{4}=-\beta \nu_{10}[12]+\varepsilon_{11}[13]$. If we take $\alpha=0, a=\nu_{01}$ then (16) is trivial for $b_{1}, b_{2}, b_{3}$ and holds for $b_{4}$ by definition of $\beta$ and since $\nu_{10} \nu_{01}=\varepsilon_{11}$. Now consider the second case in (29). By (21) $\alpha_{311}=0, x_{310} \perp \varepsilon_{10}$ (and similarly $x_{310} \perp \eta_{10}$ ), so $x_{310}=\lambda \nu_{10}$. Similarly (25) implies $x_{210}=-\beta \nu_{10}$. If $\lambda=0$ then $x_{3}=0$ and by (17) $\mathfrak{B}_{5} \subset \Phi e_{1}+\mathfrak{C}[12]$, so we may assume $\lambda=1$. Then by (20) $x_{201} \perp v_{10}$, so $x_{201} \in \Phi \varepsilon_{01}+\Phi \eta_{01}$ and by subtracting off elements of $\mathfrak{B}_{4}$ we can assume $x_{201}=0: b_{4}=v_{10}[13]-\beta v_{10}[12]$. Taking $\alpha=0, a=\varepsilon_{00}$ we obtain (16) by definition of $\beta$. Thus in all cases we get $\mathfrak{B}_{5} \subset \Phi e_{1}+\mathfrak{C}[12]$ in some isotope, and as usual by Lemma 4 even

$$
\mathfrak{B}_{5}=\Phi e_{1}+\mathfrak{C}_{\varepsilon_{11}}[12] \text { or } \mathfrak{B}_{5}=\Phi e_{1}+\varepsilon_{11} \mathfrak{\subseteq}[12] .
$$

Finally, suppose $\mathfrak{B}_{6}=\mathfrak{B}_{5}+\Phi b$. If $b=x_{2}[12]+x_{3}[13]$ then $x_{2}$ together with the $\mathfrak{\Im}_{12}$-coefficients of $\mathfrak{B}_{5}$ form a totally isotropic subspace of $\mathfrak{E}$; but the $\mathfrak{\Im}_{12}$-coefficients of $\mathfrak{B}_{5}$ already form a maximal such subspace $\mathfrak{C}_{\varepsilon_{11}}$ or $\varepsilon_{11} \mathfrak{E}$, so by subtracting off an element of $\mathfrak{B}_{5}$ we can assume $b=x_{3}[13]$. If $\mathfrak{B}_{5}=\Phi e_{1}+\mathfrak{C}_{\varepsilon_{11}}[12]$ is of first kind then $\bar{x}_{3}\left(\mathfrak{C}_{\varepsilon_{11}}\right)=0$ implies $x_{3}=0$. Thus $\mathfrak{B}_{5}=\Phi e_{1}+\mathfrak{C}_{\varepsilon_{11}}[12]$ is already maximal. If $\mathfrak{B}_{5}=\Phi e_{1}+\varepsilon_{11} \mathbb{\complement}[12]$ is of the second kind then $\bar{x}_{3}\left(\varepsilon_{11} \mathfrak{E}\right)=0$, and the only possible elements satisfying this are the $x_{3} \in \Phi \varepsilon_{11}$. Thus $\mathfrak{B}_{6}=\Phi e_{1}+\varepsilon_{11} \mathbb{E}[12]+\Phi \varepsilon_{11}[13]$ and this is maximal. These are the two types of maximal point spaces.

This completes the proof of the main theorem.

REMARKS. In case (iii), $\mathfrak{B}$ of capacity $m \geqq 2$ we saw either $\mathfrak{B}=e^{(u)} \mathfrak{\jmath} e^{(u)}$ in some isotope or $\mathfrak{B}=\left(e^{(u)}+\varepsilon f\right) \mathfrak{\Im}\left(e^{(u)}+\varepsilon f\right)$ for $e^{(u)}, f$ symmetric idempotents and $\varepsilon$ a primitive idempotent in $\mathfrak{Q}$. In the first case, if $e^{(u)}=e_{1}+\cdots+e_{m}$ we have $\operatorname{dim} \mathfrak{B}$ $=m \operatorname{dim} \mathfrak{D}_{0}+(m(m-1) / 2) \operatorname{dim} \mathfrak{D}=m+2 m(m-1)=m(2 m-1)$, while in the second, if $f=e_{n}$ we have $\operatorname{dim} \mathfrak{B}=m(2 m-1)+2 m=m(2 m+1)$. This shows that these latter $\mathfrak{B}$ cannot be obtained from a symmetric idempotent in any isotope, $\mathfrak{B} \neq e^{(v)} \mathfrak{\jmath} e^{(v)}$. Similar remarks apply in case (iii), $m=1$.

We also remark that the arguments used for $\mathfrak{D}=\Delta \oplus \Delta^{0}$ and $\mathfrak{D}=\Delta$ also apply when the capacity of $\mathfrak{\Im}$ is $n=2$.

4. The algebras $\mathfrak{A}^{+}$. In this section we consider the algebras $\mathfrak{A}^{+}$for $\mathfrak{A}$ a semisimple Artinian algebra. It is more convenient to use regularity than semisimplicity. Recall that in the Main Theorem we saw every inner ideal in $\mathfrak{A}^{+}=\mathfrak{S}\left(\mathfrak{D}_{n}\right)$, $\mathfrak{D}=\Delta \oplus \Delta^{0}$ had the form $\mathfrak{B}=g^{*} \mathfrak{S} g$ in a suitable isotope. If $g$ is idempotent in $\tilde{\mathfrak{A}}^{(u)}, g u g=g$, then $h=u g$ is idempotent in $\tilde{\mathfrak{A}}$ and satisfies $\mathfrak{B}=h^{*} \mathfrak{S} h$. Furthermore, if we write $h=f \oplus e \in \mathfrak{A} \oplus \mathfrak{A}^{0}=\tilde{\mathfrak{A}}$ then $e, f$ are idempotent in $\mathfrak{A}$ and under the isomorphism $\mathfrak{S}\left(\mathfrak{A} \oplus \mathfrak{A}^{0}\right) \rightarrow \mathfrak{A}^{+}$given by $a \oplus a \rightarrow a$ we have $h^{*}(a \oplus a) h=$ $(e \oplus f)(a \oplus a)(f \oplus e)=e a f \oplus e a f \rightarrow e a f$, so in $\mathfrak{A}^{+}$each inner ideal has the form $\mathfrak{B}=e \mathfrak{A} f$. We now give a more direct proof of this. 
THEOREM 1. If $\mathfrak{A}$ is a regular Artinian algebra then any inner ideal $\mathfrak{B}$ in $\mathfrak{A}^{+}$has the form $\mathfrak{B}=e \mathfrak{A} f$ for idempotents $e, f$.

Proof. If $\mathfrak{A}=\bigoplus \mathfrak{A}_{i}$ is a direct sum of simple Artinian ideals, by regularity any inner ideal will have the form $\mathfrak{B}=\oplus \mathfrak{B}_{i}$ for $\mathfrak{B}_{i}$ inner in $\mathfrak{A}_{i}$, so we may assume $\mathfrak{A}$ itself is simple.

Choose a pair $(e, f)$ of idempotents maximal with respect to the property $e \mathfrak{A} f \subset \mathfrak{B}$. Nonzero $e, f$ always exist-if $b \neq 0$ in $\mathfrak{B}$ then by regularity $b=b d b$ for some $d$ so that $e=b d, f=d b$ are nonzero idempotents with $e \mathfrak{A} f=b d \mathfrak{A} d b \subset b \mathfrak{A} b \subset \mathfrak{B}$. Also recall (Proposition 5) that we have the a.c.c. and d.c.c. on principal inner ideals. We claim $e^{\mathfrak{A}} f=\mathfrak{B}$, i.e. $e^{\prime} \mathfrak{B}=\mathfrak{B} f^{\prime}=0$ for $e^{\prime}=1-e, f^{\prime}=1-f$. Suppose on the contrary $b=c f^{\prime} \neq 0$ for some $c \in \mathfrak{B}$. We first show $b \in \mathfrak{B}$ : since $b \in c f^{\prime} \mathfrak{A} c f^{\prime}$ (by regularity) $\subset c f^{\prime} \mathfrak{A} c-c f^{\prime} \mathfrak{A} c f \subset c \mathfrak{A} c+c f^{\prime} \mathfrak{A} f$ where $c \mathfrak{A} c \subset \mathfrak{B}$, it suffices if $c f^{\prime} \mathfrak{A} f \subset \mathfrak{B}$. But $\mathfrak{B} \supset\left\{c f^{\prime} \mathfrak{A} e \mathfrak{A} f\right\}=c f^{\prime} \mathfrak{A} e \mathfrak{A} f\left(\right.$ since $\left.f f^{\prime}=0\right)=c f^{\prime} \mathfrak{X} f($ by simplicity and $e \neq 0)$. Thus $b=c f^{\prime} \in \mathfrak{B}$; if $b=b d b$ we may assume $d=f^{\prime} d$, so that $f f^{\prime}=f^{\prime} f=0$ gives $f d=b f=0$ and therefore the nonzero idempotent $g=d b$ has $f g=g f=0$ and hence $f+g>f$. On the other hand $\mathfrak{B}$ contains $\{e \mathfrak{A} f f \mathfrak{A} b\}=e \mathfrak{A} f \cdot f \mathfrak{A} \cdot b($ since $b f=0)=e \mathfrak{A} f \mathfrak{A} b=e \mathfrak{A} b$ (by simplicity and $f \neq 0) \supset e \mathfrak{A} g$ so $\mathfrak{B} \supset e \mathfrak{A}(f+g)$ contradicts the maximality of $f$. Thus $\mathfrak{B} f^{\prime}=0$, and similarly $e^{\prime} \mathfrak{B}=0$, so $\mathfrak{B}=e \mathfrak{A} f$.

COROllary. An inner ideal $\mathfrak{B}$ in a regular Artinian algebra $\mathfrak{A}^{+}$satisfies $\mathfrak{B} \mathfrak{A} \mathfrak{B} \subset \mathfrak{B}$ (in particular, it is a subalgebra), and it is the intersection of a left and a right ideal: $\mathfrak{B}=\mathfrak{B} \mathfrak{A} \cap \mathfrak{A} \mathfrak{B}$.

From this we see that not only does $\mathfrak{A}^{+}$have the a.c.c. and d.c.c. on all inner ideals, we can actually get a bound on the length of any chain of inner ideals.

COROllary. A regular Artinian algebra has the a.c.c. and d.c.c. on all inner ideals. If $\mathfrak{A}$ has capacity $n$ then any proper chain $\mathfrak{A}^{+}=\mathfrak{B}_{1}>\mathfrak{B}_{2}>\cdots>\mathfrak{B}_{r}>0$ of inner ideals has length $r \leqq 2 n-1$.

Proof. We have decreasing chains $\mathfrak{A} \mathfrak{B}_{i}, \mathfrak{B}_{i} \mathfrak{A}$ of left and right ideals, so we may find decreasing chains $1=e_{1} \geqq e_{2} \geqq \cdots \geqq e_{r} \geqq 0$ and $1=f_{1} \geqq f_{2} \geqq \cdots \geqq f_{r} \geqq 0$ of nonzero idempotents with $\mathfrak{A} \mathfrak{B}_{i}=\mathfrak{A} f_{i}, \mathfrak{B}_{i} \mathfrak{A}=e_{i} \mathfrak{A}$. Then $\mathfrak{B}_{i}=\mathfrak{B}_{i} \mathfrak{A} \cap \mathfrak{A} \mathfrak{B}_{i}=e_{i} \mathfrak{A} \cap \mathfrak{A} f_{i}=e_{i} \mathfrak{A} f_{i}$. Since $\mathfrak{B}_{i}>\mathfrak{B}_{i+1}$ we have either $e_{i}>e_{i+1}$ or $f_{i}>f_{i+1}$. If $c(e)$ denotes the capacity (or rank) and we set $c\left(\mathfrak{B}_{i}\right)=c\left(e_{i}\right)+c\left(f_{i}\right)$ then $c\left(\mathfrak{B}_{i}\right)>c\left(\mathfrak{B}_{i+1}\right)$. As $c\left(\mathfrak{B}_{1}\right)=c(1)+c(1)=2 n$ and $c\left(\mathfrak{B}_{r}\right) \geqq 2$ and the capacity at the $r$ th stage $\mathfrak{B}_{r}$ is at most $c\left(\mathfrak{B}_{1}\right)-(r-1)$, we must have $2 n+1-r \geqq 2$ or $2 n-1 \geqq r$.

5. The algebras $\mathfrak{S}\left(\mathfrak{A},{ }^{*}\right)$. We begin by obtaining geometric representations of these algebras and verifying thereby that algebras of types IV and V in our classification cannot be isotopic.

First we consider algebras of the form $\mathfrak{J}=\mathfrak{S}_{\mathfrak{E}}\left(\Delta_{n}, \Delta_{0}, \gamma\right)$ where (i) $\Delta$ is an associative division algebra with involution $\delta \rightarrow \delta$, (ii) $\Delta_{0}$ is an ample subspace (i.e. $\Delta_{0} \subset \mathfrak{S}\left(\Delta,{ }^{-}\right)$contains 1 and $\delta \Delta_{0} \delta \subset \Delta_{0}$ for all $\delta \in \Delta$-this implies it contains all 
norms $\delta \delta$ and traces $\delta+\delta$ ), and (iii) the $\gamma_{i}$ are invertible elements of $\Delta_{0}$. $\Im$ is an ample outer ideal in $\tilde{\mathfrak{J}}=\mathfrak{H}\left(\mathfrak{A},{ }^{*}\right)$ for $\mathfrak{A}=\Delta_{n}$ under the involution $x^{*}=\gamma^{-1} \bar{x}^{t} \gamma$, which consists of those *-symmetric matrices $x=\left(\alpha_{i j}\right)$ whose diagonal entries $\alpha_{i i}$ lie in $\gamma_{i}^{-1} \Delta_{0}$. (A general *-symmetric matrix need only have $\alpha_{i i}=\gamma_{i}^{-1} \bar{\alpha}_{i i} \gamma_{i}$, i.e.

$$
\gamma_{i} \alpha_{i i}=\bar{\alpha}_{i i} \bar{\gamma}_{i}=\overline{\gamma_{i} \alpha_{i i}}
$$

is symmetric and $\alpha_{i i} \in \gamma_{i}^{-1} \mathfrak{S}(\Delta,-)$.)

Geometrically, $\mathfrak{A}=\operatorname{End}_{\Delta} \mathfrak{B}$ for $\mathfrak{B}$ an $n$-dimensional right vector space over $\Delta$ with basis $v_{1}, \ldots, v_{n}$ where the matrix $x=\left(\alpha_{i j}\right)$ corresponds to the linear transformation $x\left(v_{i}\right)=\sum v_{j} \alpha_{j i}$. The involution $*$ corresponds to the adjoint relative to the nondegenerate (and nonalternate) Hermitian form $\langle v, w\rangle$ on $\mathfrak{B}$ defined by $\left\langle v_{i}, v_{i}\right\rangle$ $=\gamma_{i},\left\langle v_{i}, v_{j}\right\rangle=0(i \neq j)$; necessarily all "quadratic values" $\langle v, v\rangle$ lie in $\Delta_{0}$. Here $\mathfrak{S}$ corresponds to the algebra $\mathfrak{S}\left(\langle\rangle,, \Delta_{0}\right)$ of those selfadjoint transformations $x$ such that the values $\langle v, x(v)\rangle$ still lie in $\Delta_{0}$. Conversely, any nondegenerate and nonalternate Hermitian form on $\mathfrak{B}$ can be diagonalized, and relative to an orthogonal basis with $\left\langle v_{i}, v_{i}\right\rangle=\gamma_{i}$ we have a ${ }^{*}$-isomorphism of $\left(\operatorname{End}_{\Delta} \mathfrak{B},{ }^{*}\right)$ with $\left(\Delta_{n}, *(\gamma)\right)$; if the $\gamma_{i}$ belong to an ample subspace $\Delta_{0}$ we get an isomorphism of $\mathfrak{S}\left(\langle\rangle,, \Delta_{0}\right)$ with the matrix algebra $\mathfrak{S}\left(\Delta_{n}, \Delta_{0}, \gamma\right)$ since the condition $\left\langle v_{i}, x\left(v_{i}\right)\right\rangle$ $\in \Delta_{0},\left\langle v_{i}, x\left(v_{j}\right)\right\rangle+\left\langle v_{j}, x\left(v_{i}\right)\right\rangle \in \Delta_{0}$ that $x=x^{*}$ belong to $\mathfrak{S}\left(\langle\rangle,, \Delta_{0}\right)$ reduces to $\gamma_{i} \alpha_{i i} \in \Delta_{0}, \gamma_{i} \alpha_{i j}+\gamma_{j} \alpha_{j i} \in \Delta_{0}$. But the latter is a trace,

$$
\gamma_{i} \alpha_{i j}+\gamma_{j} \alpha_{j i}=\gamma_{i} \alpha_{i j}+\gamma_{j}\left(\gamma_{j}^{-1} \bar{\alpha}_{i j} \gamma_{i}\right)=\gamma_{i} \alpha_{i j}+\overline{\gamma_{i} \alpha_{i j}} \text {, }
$$

and hence automatically belongs to $\Delta_{0}$, so the only condition is that $\alpha_{i i} \in \gamma_{i}^{-1} \Delta_{0}$. Thus we may represent the abstract Jordan algebra $\mathfrak{I}$ either as an algebra $\mathfrak{S}\left(\Delta_{n}, \Delta_{0}, \gamma\right)$ of matrices or as an algebra $\mathfrak{S}\left(\langle\rangle,, \Delta_{0}\right)$ of linear transformations.

The other kind of algebra we must consider is $\mathfrak{I}=\mathfrak{S}_{\mathfrak{C}}\left(\mathfrak{Q}_{n}, \mathfrak{Q}_{0}, \gamma\right)$ for $\mathfrak{Q}$ a split quaternion algebra, $\mathfrak{Q}_{0}=\Phi$ the center of $\mathfrak{Q}$. In this case we may choose a different coordinatization so that $\gamma=1$, and $\mathfrak{J}=\mathfrak{S}\left(\mathfrak{Q}_{n}, \Phi\right)$. (Note that in characteristic $\neq 2$ $\mathfrak{Q}_{0}=\Phi=\mathfrak{S}(\mathfrak{Q})$ consists of all symmetric elements of $\mathfrak{Q}$ under the standard involution, but in characteristic $2 \mathfrak{Q}_{0}=\Phi$ is a proper ample subspace of $\mathfrak{Q}$.) Since $\mathfrak{Q}$ is split we have $\mathfrak{Q}=\Phi_{2}$, and we have a ${ }^{*}$-isomorphism of $\left(\mathfrak{Q}_{n},{ }^{*}\right)$ with $\left(\Phi_{2 n},{ }^{*}(s)\right)$ for ${ }^{*}(s)$ the canonical involution $x^{*(s)}=s^{-1} \bar{x}^{t} s$ determined by the standard symplectic $(2 n) \times(2 n)$ matrix

$$
s=\left(\begin{array}{cc}
0 & I_{n} \\
-I_{n} & 0
\end{array}\right)
$$

where the mapping $\mathfrak{Q}_{n} \rightarrow \Phi_{2 n}$ sends $\varepsilon_{i j} e_{k l} \rightarrow e_{k+i n, l+j n}$ for $\varepsilon_{i j}(0 \leqq i, j \leqq 1)$ and $e_{k l}(1 \leqq k, l \leqq n)$ matrix units for $\mathfrak{Q}$ and $\Phi_{n}$ respectively. Thus $\mathfrak{H}\left(\mathfrak{Q}_{n}\right)$ is isomorphic to $\mathfrak{S}\left(\Phi_{2 n},{ }^{*}(s)\right)$. The elements of $\mathfrak{S}\left(\mathfrak{Q}_{n}, \Phi\right)$ are precisely those ${ }^{*}$-symmetric matrices with diagonal entries in which $\varepsilon_{01}, \varepsilon_{10}$ do not appear, and since $\varepsilon_{01} e_{i i} \rightarrow e_{i, i+n}$, $\varepsilon_{10} e_{i i} \rightarrow e_{i+n, i}$ this corresponds to those matrices $x=\left(\alpha_{i j}\right)$ in $\mathfrak{S}\left(\Phi_{2 n},{ }^{*}(s)\right)$ in which $e_{i, i+n}$ and $e_{i+n, i}$ do not appear: $\alpha_{i, i+n}=\alpha_{i+n, i}=0$. We denote this algebra by $\mathfrak{S}\left(\Phi_{2 n}, 0,{ }^{*}(s)\right)$ (in characteristic $\neq 2$ it coincides with $\mathfrak{S}\left(\Phi_{2 n},{ }^{*}(s)\right)$ ). 
To interpret this geometrically we introduce a $2 n$-dimensional vector space $\mathfrak{B}$ with basis $v_{1}, \ldots, v_{2 n}$ over $\Phi$ and a nondegenerate alternate bilinear form $\langle v, w\rangle$ on $\mathfrak{B}$ with $\left\langle v_{i}, v_{i+n}\right\rangle=-\left\langle v_{i+n}, v_{i}\right\rangle=1(1 \leqq i \leqq n)$ and all other products zero. Then $\left(\Phi_{2 n},{ }^{*}(s)\right)$ is ${ }^{*}$-isomorphic to $\left(\mathfrak{A},{ }^{*}\right)$ for $\mathfrak{A}=\operatorname{End}_{\Phi} \mathfrak{B}$ and ${ }^{*}$ the adjoint relative to the form $\langle v, w\rangle$. Under this isomorphism the elements of $\mathfrak{S}_{2}\left(\Phi_{2 n}, 0, *(s)\right)$ correspond to the alternate transformations $x,\langle v, x(v)\rangle=0$ for all $v \in \mathfrak{B}$ (in analogy with the situation in $\left.\mathfrak{S}\left(\Delta_{n}, \Delta_{0}, \gamma\right)\right)$. Indeed, a transformation $x$ with matrix $\left(\alpha_{i j}\right)$ is alternate if and only if $\left\langle v_{i}, x\left(v_{i}\right)\right\rangle=\left\langle v_{i}, x\left(v_{j}\right)\right\rangle+\left\langle v_{j}, x\left(v_{i}\right)\right\rangle=0$ or

$$
\begin{aligned}
\alpha_{i, i+n} & =\alpha_{i+n, i}=\alpha_{i+n, j}+\alpha_{j+n, i}=\alpha_{i, j+n}+\alpha_{j, i+n} \\
& =\alpha_{i j}-\alpha_{j+n, i+n}=0 \quad(1 \leqq i, j \leqq n)
\end{aligned}
$$

which is just the condition that $\left(\alpha_{i j}\right)$ belong to $\mathfrak{S}\left(\Phi_{2 n}, 0, *(s)\right)$. We denote the (Jordan) algebra of such transformations by Alt $(\langle\rangle$,$) . Conversely, given any$ nondegenerate alternate form $\langle v, w\rangle$ on $\mathfrak{B}$ we can find a symplectic basis $v_{1}, \ldots, v_{2 n}$, and such a basis determines an isomorphism of Alt $(\langle\rangle$,$) with \mathfrak{S}\left(\Phi_{2 n}, 0, *(s)\right)$. Thus we have isomorphisms

$$
\mathfrak{\Im} \cong \mathfrak{S C}_{\mathfrak{g}}\left(\mathfrak{Q}_{n}, \Phi\right) \cong \mathfrak{S}\left(\Phi_{2 n}, 0,{ }^{*}(s)\right) \cong \operatorname{Alt}(\langle,\rangle) .
$$

Let us make one more remark about Alt $(\langle\rangle$,$) . We claim it is uniquely deter-$ mined by $\mathfrak{A}=\operatorname{End}_{\Phi} \mathfrak{B}$ and the involution *, not by how we represent $\mathfrak{A}$ and $*$ in terms of $\mathfrak{B}$ and $\langle$,$\rangle . Indeed, Alt (\langle\rangle$,$) is the unique minimal ample outer ideal in$ $\mathfrak{S}(\mathfrak{A}, *)$ and consists of all traces $t(a)=a+a^{*}$ for $a \in \mathfrak{A}$. If Alt $(\mathfrak{H}, *)$ denotes the set of traces, it forms a subspace of $\mathfrak{S}\left(\mathfrak{A},{ }^{*}\right)$ containing $1=\sum_{1}^{n}\left(e_{i i}+e_{i+n, i+n}\right)$ $=\sum_{1}^{n}\left(e_{i i}+e_{i i}^{*}\right)=t\left(\sum_{1}^{n} e_{i i}\right)$ and closed under outer multiplication $b t(a) b^{*}=b a b^{*}$ $+b a^{*} b^{*}=t\left(b a b^{*}\right)$, hence is an ample outer ideal. As any ample outer ideal contains norms and traces, Alt $\left(\mathfrak{A},{ }^{*}\right)$ is the unique minimal one. But it contains Alt $(\langle\rangle$, since any alternate $x=\left(\alpha_{i j}\right)=\sum \alpha_{i j} e_{i j}$ satisfying the above relations on the coefficients $\alpha_{i j}$ is a trace $x=t(a)$ for

$$
a=\sum_{i, j} \alpha_{i j} e_{i j}+\sum_{i<j} \alpha_{i, j+n} e_{i, j+n}+\sum_{i<j} \alpha_{i+n, j} e_{i+n, j} \quad(1 \leqq i, j \leqq n) .
$$

Thus Alt $(\langle\rangle)=$, Alt $\left(\mathfrak{A},{ }^{*}\right)$.

We now consider isotopes of the algebras $\mathfrak{S}_{2}\left(\Delta_{n}, \Delta_{0}, \gamma\right)$ and $\mathfrak{S}\left(\mathfrak{Q}_{n}, \Phi\right)$. We use the geometric representation of these as $\mathfrak{S}\left(\langle\rangle,, \Delta_{0}\right)$ and Alt $(\langle\rangle$,$) for \langle v, w\rangle$ a Hermitian or alternate bilinear form on a vector space $\mathfrak{B}$. In either case the algebra is an ample outer ideal in $\mathfrak{S}\left(\mathfrak{A},{ }^{*}\right)$ for $\mathfrak{A}=$ End $\mathfrak{B}$ and ${ }^{*}$ the adjoint relative to the bilinear form. If $u=u^{*}$ is invertible in $\mathfrak{A}$ then the map $\varphi(x)=x u$ affords a *-isomorphism of $\left(\mathfrak{Q}^{(u)},{ }^{*}\right)$ with $\left(\mathfrak{A},{ }^{*}(u)\right)$ for $x^{*(u)}=u^{-1} x^{*} u\left(u^{*}=u\right.$ guarantees * remains an involution on the associative isotope $\left.\mathfrak{A}^{(u)}\right)$. In fact, $\varphi\left(x \circ_{u} y\right)=\varphi(x u y)$ $=x u y u=\varphi(x) \varphi(y)$ so $\mathfrak{A}^{(u)} \rightarrow \mathfrak{A}$ is an algebra isomorphism, and $\varphi\left(x^{*}\right)=x^{*} u$ $=u^{-1} u^{*} x^{*} u=u^{-1}(x u)^{*} u=\varphi(x)^{*(u)}$ so it is a ${ }^{*}$-isomorphism. This induces an isomorphism of $\mathfrak{S}\left(\mathfrak{A},{ }^{*}\right)^{(u)}$ with $\mathfrak{S}\left(\mathfrak{A},{ }^{*}(u)\right)$. If ${ }^{*}$ is the transpose relative to a non- 
degenerate form $\langle v, w\rangle$ on $\mathfrak{B}$ then $*(u)$ is the transpose relative to $\langle v, w\rangle^{(u)}$ $=\langle v, u(w)\rangle$ since

$$
\begin{aligned}
\langle v, x(w)\rangle^{(u)} & =\langle v, u(x(w))\rangle=\left\langle x^{*} u(v), w\right\rangle \\
& =\left\langle u^{-1} x^{*} u(v), u(w)\right\rangle=\left\langle x^{*(u)}(v), w\right\rangle^{(u)} .
\end{aligned}
$$

Here $\langle v, w\rangle^{(u)}$ is nondegenerate if $\langle v, w\rangle$ is because $u$ is invertible. If $\langle v, w\rangle$ is Hermitian or skew-symmetric so is $\langle v, w\rangle^{(u)}$ since $u^{*}=u$. If the values $\langle v, v\rangle$ all lie in $\Delta_{0}$ and $u \in \mathfrak{F}\left(\langle\rangle,, \Delta_{0}\right)$ then the values $\langle v, v\rangle^{(u)}=\langle v, u(v)\rangle$ still lie in $\Delta_{0}$, and if $\langle v, w\rangle$ is alternate and $u \in \operatorname{Alt}(\langle\rangle$,$) then \langle v, w\rangle^{(u)}$ will again be alternate. If $\langle v, w\rangle$ is Hermitian but nonalternate then $\langle v, w\rangle^{(u)}$ will again be nonalternate unless $\Delta$ is a field of characteristic 2 with identity involution, $\mathfrak{B}$ is even dimensional, and $u$ is an alternate transformation. (It is well known that an alternate sesquilinear form must have $\Delta$ a field with identity involution, and if it is also Hermitian then $\Delta$ must have characteristic 2.)

We say that an involution on $\mathfrak{A}=$ End $\mathfrak{B}$ is a diagonal involution if it can be represented as the adjoint relative to a nondegenerate nonalternate Hermitian form, and symplectic or alternate if it can be represented as the adjoint relative to a nondegenerate alternate form. Thus a diagonal involution may have an isotope which is alternate (witness the $s$-isotope of the transpose involution on $\Phi_{2 n}$ in characteristic 2). However, even if $\langle v, w\rangle^{(u)}$ is alternate the algebra $\mathfrak{S}\left(\langle,\rangle, \Delta_{0}\right)^{(u)}$ $=\mathfrak{S}\left(\langle,\rangle^{(u)}, \Delta_{0}\right)$ is not the same as Alt $\left(\langle,\rangle^{(u)}\right)=\mathfrak{S}\left(\langle,\rangle^{(u)}, 0\right)$ since the former contains some nonalternate transformations.

In the Main Theorem we saw that for Artinian $\mathfrak{A}$ with diagonal involution every inner ideal was principal, $\mathfrak{B}=b \Im b$, and in a suitable isotope $\mathfrak{B}=e^{(u)} \mathfrak{\jmath} e^{(u)}$. As before, the fact that $e^{(u)}$ is idempotent in $\Im^{(u)}, e^{(u)}=e^{(u)} u e^{(u)}$, implies $e=u e^{(u)}$ is idempotent in $\mathfrak{A}$ itself and $e^{*} \mathfrak{\Im} e=e^{(u)} u \mathfrak{\Im} u e^{(u)}=e^{(u)} \mathfrak{\Im} e^{(u)}=\mathfrak{B}$. Thus we actually have $\mathfrak{B}=e^{*} \mathfrak{J} e$ for some idempotent in $\mathfrak{A}$ (not necessarily in $\mathfrak{\Im}=\mathfrak{S}\left(\mathfrak{A},{ }^{*}\right)$ ).

THEOREM 2. Every inner ideal of $\mathfrak{\Im}=\mathfrak{S}_{\mathfrak{C}}\left(\Delta_{n}, \Delta_{0}, \gamma\right)$ is principal, $\mathfrak{B}=b \Im b$. An alternate description is that $\mathfrak{B}=e^{*}$ ฐe for some idempotent $e$ in $\Delta_{n}$.

Proof. We use the geometric representation $\mathfrak{I}=\mathfrak{S}\left(\langle\rangle,, \Delta_{0}\right)$. Say that a transformation $x$ is alternate if $\langle v, x(v)\rangle=0$ for all $v$. We need a

LEMMA 5. If $\langle v, w\rangle$ is a nonalternate Hermitian form on $\mathfrak{B}$ and $\mathfrak{\Im}$ an ample outer ideal in $\mathfrak{S}\left(\mathfrak{A},{ }^{*}\right)$ then the only element $x \in \mathfrak{A}$ such that all elements of $x^{*} \mathfrak{\Im} x$ are alternate, $\left\langle v, x^{*} \Im x(v)\right\rangle=0$ for all $v$, is $x=0$. In particular, $x^{*} \mathfrak{\Im} x=0$ implies $x=0$.

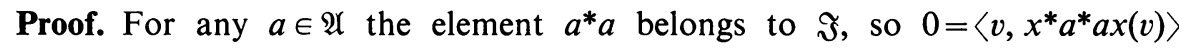
$=\langle a x(v), a x(v)\rangle$. If $x \neq 0$ some $x(v) \neq 0$, in which case for any $w \in \mathfrak{B}$ there is $a \in \mathfrak{A}$ sending $x(v)$ to $w, a(x(v))=w$. But this would imply $\langle w, w\rangle=0$ for all $w$, contradicting our assumption that the form is nonalternate. Thus we must have $x=0$.

Returning to the proof of the theorem, the Lemma guarantees that any inner ideal $\mathfrak{B} \neq 0$ contains nonalternate elements (if $b \neq 0$, taking $x=b$ gives such elements in $b \mathfrak{\Im} b \subset \mathfrak{B})$. 
Among the principal inner ideals $b \Im b$ contained in $\mathfrak{B}$ for $b$ nonalternate, choose a maximal one (by Propositions 4, $6 \mathfrak{\Im}$ has the a.c.c. and d.c.c. on principal inner ideals). By regularity (Propositions 4, 6 again) $b=b d b$, so $e=d b$ and $e^{*}=b d$ are idempotents in $\mathfrak{A}$ (not necessarily in $\mathfrak{\Im}$ ), and $e^{*} \mathfrak{\Im} e=b d \mathfrak{J} d b \subset b \mathfrak{\Im} b$. Moreover, we have equality $b \Im b=e^{*} \mathfrak{\Im} e$ since $b \Im b=e^{*} b \Im b e \subset e^{*} \mathfrak{\jmath} e$. We will show that $\mathfrak{B}=e^{*} \mathfrak{\jmath} e$ $=b \Im b$.

For any $c \in \mathfrak{B}$ we have $e^{*} c+c e=b d c+c d b=\{b d c\} \in \mathfrak{B}$, hence for $e^{\prime}=1-e$ also

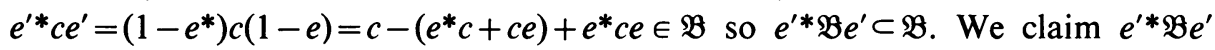
$=0$. If $c \in e^{*} \mathfrak{B} e^{\prime}$ were nonzero then the element $b^{\prime}=b+c \in \mathfrak{B}$ would be nonalternate since $\left\langle e v, b^{\prime} e(v)\right\rangle=\left\langle v, e^{*} b^{\prime} e(v)\right\rangle=\langle v, b(v)\rangle \neq 0$ by choice of $b$ (note $\left.b=e^{*} b=b e, e^{*} c=c e=0\right)$. The principal inner ideal $b^{\prime} \mathfrak{J} b^{\prime} \subset \mathfrak{B}$ contains $b^{\prime} e \mathfrak{a} e^{*} b^{\prime}$ $=b \Im b$ and also $b^{\prime} e^{\prime} \Im e^{* *} b^{\prime}=c \Im c$, which by regularity contains $c \notin b \Im b=e^{*} \Im e$, so

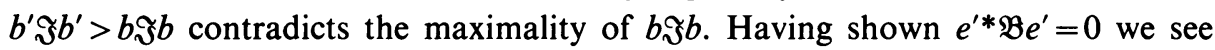
$e^{* *}\left(c e \Im e^{*} c\right) e^{\prime}=0$ for any $c \in \mathfrak{B}$, and by the lemma $x^{*} \Im x=0$ for $x=e^{*} c e^{\prime}$ implies $x=0$. Also $x^{*}=0$, so $e^{*} c e^{\prime}=e^{*} c e=0$. This shows that for any $c \in \mathfrak{B}$ we have $c=\left(e^{*}+e^{*}\right) c\left(e+e^{\prime}\right)=e^{*} c e$ and hence $\mathfrak{B}=e^{*} \mathfrak{g} e$.

Rephrasing this in abstract terms,

THEOREM 3. If $\mathfrak{A}$ is a regular Artinian algebra with diagonal involution, and $\mathfrak{\Im}$ is an ample outer ideal in $\mathfrak{E}\left(\mathfrak{U},{ }^{*}\right)$, then any inner ideal $\mathfrak{B}$ in $\mathfrak{\Im}$ is principal: $\mathfrak{B}=b \mathfrak{\Im} b$. We can also express $\mathfrak{B}$ as $\mathfrak{B}=e^{*} \mathfrak{\jmath}$ e for some idempotent $e \in \mathfrak{A}$.

CoRollaRY. Any inner ideal in $\mathfrak{J}$ is the intersection $\mathfrak{B}=\mathfrak{J} \cap \tilde{\mathfrak{B}}$ for $\tilde{\mathfrak{B}}$ an inner ideal in $\mathfrak{A}$. If $b, c$ are in $\mathfrak{B}$ then $b a c+c a^{*} b \in \mathfrak{B}$ for any $a \in \mathfrak{A}$.

COROLlaRY. If $\mathfrak{A}$ is a regular Artinian algebra with diagonal involution, then any ample outer ideal $\mathfrak{\Im}$ in $\mathfrak{S}\left(\mathfrak{A}, *^{*}\right)$ has the a.c.c. and d.c.c. on all inner ideals. If $\mathfrak{A}$ has capacity $n$ then any proper chain $\mathfrak{\Im}=\mathfrak{B}_{1}>\mathfrak{B}_{2}>\cdots>\mathfrak{B}_{r}>0$ of inner ideals in $\mathfrak{\Im}$ has length $r \leqq n$.

Proof. If we are given inner ideals $\mathfrak{B}=e^{*} \Im e \supset f^{*} \Im f=\mathfrak{C}$ we can renormalize so that $e \geqq f$ : we have $\mathfrak{C}=c \mathfrak{\Im}$ for some $c$, and regularity $c=c d c \in \mathfrak{C} \subset e^{*} \mathfrak{\Im} e$ implies $e^{*} c=c e=c$, so if we set $g=e d c$ we have $g^{2}=e d c e d c=e d c d c=e d c=g, g^{*} \Im g$ $=c d e^{*} \Im e d c \subset c \Im c=\mathfrak{夭}$, and $\mathfrak{夭}=c \Im c=c d(c \Im c) d c \subset c d \mathfrak{B} d c=c d e^{*} \Im e d c=g^{*} \Im g$, so that $\mathfrak{E}=g^{*} \mathfrak{I} g$ where the idempotent $g$ has $e \geqq g$ since $e g e=e^{2} d c e=e d c=g$.

Given $\mathfrak{I}=\mathfrak{B}_{1}>\cdots>\mathfrak{B}_{r}>0$ we can write $\mathfrak{B}_{i}=e_{i}^{*} \mathfrak{S} e_{i}$ for $1=e_{1}>e_{2}>\cdots>e_{r}>0$ and hence $r \leqq n$.

Turning to the case of alternate involutions, since $\mathfrak{\Im}=\mathfrak{S}\left(\Phi_{2 n},{ }^{*}(s)\right)$ is finite dimensional over its center $\Phi$, by Proposition 2 all inner ideals are $\Phi$-subspaces and we have the a.c.c. and d.c.c.

THEOREM 4. If $\mathfrak{A}$ is a regular Artinian algebra with alternate involution, then $\mathfrak{\Im}=$ Alt $\left(\mathfrak{A},{ }^{*}\right)$ has the a.c.c. and d.c.c. on all inner ideals. 
We saw in the Main Theorem that the inner ideals were either point spaces or had the form $e^{(u)} \mathfrak{\Im} e^{(u)}$ in some isotope, hence as before the form $e^{*} \mathfrak{\Im} e$ for $e$ idempotent in $\mathfrak{A}$. We give a variant proof.

THEOREM 5. If $\mathfrak{A}$ is a regular Artinian algebra with alternate involution, then any inner ideal $\mathfrak{B}$ in $\mathfrak{\Im}=\mathrm{Alt}\left(\mathfrak{A},{ }^{*}\right)$ is either a point space or of the form $e^{*} \mathfrak{\Im}$. A maximal point space either has the form $\mathfrak{B}=(e+f)^{*} \mathfrak{\Im}(e+f)$ or $\mathfrak{B}=e^{*} \mathfrak{\jmath} e+t\left(f \mathfrak{A} e^{\prime}\right)$ for e $a$ rank 2 idempotent, $f$ a rank 1 idempotent satisfying $e f=f e=0$ or $e^{*} f=f e^{*}=f$ respectively.

Proof. We begin as in the case of a diagonal involution, choosing a maximal principal inner ideal $b \mathfrak{\Im} b \subset \mathfrak{B}$; if $\mathfrak{B}$ is not a point space we can assume $b \Im b \neq \Phi b$, otherwise assume $\mathfrak{B}$ is a maximal point space. We have $b \Im b=e^{*} \mathfrak{\jmath} e$ for $e=d b$, $e^{*}=b d$ where $b=b d b$, and $e^{* *} \mathfrak{B} e^{\prime}=0$ as before. We also obtain $e^{* *} c e^{\Im} e^{*} c e^{\prime}=0$ for any $c \in \mathfrak{B}$; however, unlike the diagonal case, $x^{*} \mathfrak{\Im} x=0$ does not imply $x=0$.

LEMma 6. If $\langle v, w\rangle$ is a nondegenerate alternate form on $\mathfrak{B}$ and $\langle\mathfrak{J}, w\rangle=0$ for $v, w \neq 0, \Im=$ Alt $(\langle\rangle$,$) , then w \in \Phi v$. Consequently $\Im v=v^{\perp}$ for any $v \neq 0$, while if $\mathfrak{W}$ is a subspace of dimension $>1$ then $\mathfrak{\Im W}=\mathfrak{B}$. A transformation $x$ satisfies $x * \mathfrak{I} x=0$ if and only if $x$ has rank $\leqq 1$.

Proof. Since $\mathfrak{\Im}$ contains all $a+a^{*}$ for $a \in \mathfrak{A}=$ End $\mathfrak{B}$, if $w$ were independent of $v$ we could find $a \in \mathfrak{A}$ with $a(w)=0$ but $\langle a(v), w\rangle \neq 0$. But then $\langle a(v), w\rangle=\langle a(v), w\rangle$ $+\langle v, a(w)\rangle=\left\langle\left(a+a^{*}\right) v, w\right\rangle=0$, a contradiction. Thus $w$ must lie in $\Phi v$. From this we see $(\Im v)^{\perp}=\Phi v$ if $v \neq 0$, hence $\Im v=(\Im v)^{\perp \perp}=v^{\perp}$. If $\mathfrak{W}$ has dimension $>1$ then $\mathfrak{S} \mathfrak{B}=\sum_{w \in \mathfrak{W}} \mathfrak{W} w=\sum_{w \in \mathfrak{W}} w^{\perp}=\mathfrak{B}$ (any $w^{\perp}$ has codimension 1, and if $w_{1}^{\perp}=w_{2}^{\perp}$ then $w_{1} \in \Phi w_{2}$ so two independent $w_{i}^{\perp}$ will span $\left.\mathfrak{B}\right)$. If $x^{*} \mathfrak{\Im} x=0$ then $0=\left\langle x^{*} \mathfrak{F} x(\mathfrak{B}), \mathfrak{B}\right\rangle$ $=\langle\mathfrak{I} x(\mathfrak{B}), x(\mathfrak{B})\rangle$ implies $\mathfrak{I} x(\mathfrak{B}) \neq \mathfrak{B}$, so by the above $x(\mathfrak{B})$ has dimension $\leqq 1$.

Returning to the proof of the theorem, this says $x=e^{*} c e^{\prime}$ has rank $\leqq 1$ for all $c \in \mathfrak{B}$. If $x=0$ then $e^{*} c e^{\prime}=e^{*} c e=e^{* *} c e^{\prime}=0$ for all $c, c=e^{*} c e$, and $\mathfrak{B}=e^{*} \mathfrak{J} e=b \Im b$ so we are done. Thus we may assume some $c_{10}=e^{*} c e^{\prime} \neq 0$; by the lemma $c_{10}(\mathfrak{B})$ $=\Phi v_{0}$ is 1-dimensional. If $c_{10}^{\prime}=e^{*} c^{\prime} e^{\prime}$ for some other $c^{\prime} \in \mathfrak{B}$ then

$$
0=e^{*}\left\{c^{\prime}\left(e \Im e^{*}\right) c+c\left(e \Im e^{*}\right) c^{\prime}\right\} e^{\prime}=c_{10}^{*} \Im c_{10}+c_{10}^{*} \Im c_{10}^{\prime} .
$$

Choose $v \in \mathfrak{B}$ so $c_{10}(v)=v_{0}$; for any $w \in \operatorname{Ker} c_{10}$ we have

$$
0=\left\langle\left\{c_{10}^{\prime *} \Im c_{10}+c_{10}^{*} \Im c_{10}^{\prime}\right\} v, w\right\rangle=\left\langle\Im c_{10}(v), c_{10}^{\prime}(w)\right\rangle
$$

so by the lemma $c_{10}^{\prime}(w) \in \Phi c_{10}(v)=\Phi v_{0}$. Either $c_{10}^{\prime}(w)=0$ for all $w \in \operatorname{Ker} c_{10}$, in which case $\operatorname{Ker} c_{10} \subset \operatorname{Ker} c_{10}^{\prime}$ and therefore $\operatorname{Ker} c_{10}^{\prime}=\operatorname{Ker} c_{10}$ since both have codimension 1, or else some $c_{10}^{\prime}(w) \neq 0$, in which case Range $c_{10}^{\prime}=\Phi c_{10}^{\prime}(w)$ $\subset$ Range $c_{10}$ implies Range $c_{10}^{\prime}=$ Range $c_{10}$ since both have dimension 1. As usual, this linear choice for each $c_{10}^{\prime}$ implies either $\operatorname{Ker} c_{10}^{\prime}=\operatorname{Ker} c_{10}$ for all $c_{10}^{\prime}$ or else Range $c_{10}^{\prime}=$ Range $c_{10}$ for all $c_{10}^{\prime}$. These two cases lead to two kinds of inner ideals.

Consider the case when all $c_{10}^{\prime}$ have the same kernel. If $c_{10} w c_{10}=c_{10}$ by regularity 
$\left(w=e^{\prime} w e^{*}\right)$ then $f=w c_{10}$ is an idempotent orthogonal to $e\left(e^{\prime} f=f e^{\prime}=f\right)$ and of rank 1 ; then $f^{*}$ is also of rank 1 , so by the lemma

$$
f \Im f^{*}=f^{*} \Im f=0
$$

Furthermore, $\operatorname{Ker} f=\operatorname{Ker} c_{10}: \operatorname{Ker} f \supset \operatorname{Ker} c_{10}$ and they both have codimension 1. Thus for any $c^{\prime} \in \mathfrak{B}$ we have $c_{10}^{\prime}=c_{10}^{\prime} f\left(c_{10}^{\prime}=e^{*} c e^{\prime}\right)$; as $e^{\prime *} c e^{\prime}=0$ we see $c^{\prime}=e^{*} c e$ $+e^{*} c e^{\prime}+e^{*} c e=e^{*} c e+t\left(e^{*} c e^{\prime}\right)=e^{*} c e+t\left(e^{*} c f\right)$,

$$
\mathfrak{B} \subset \mathfrak{B}^{\prime}=e^{*} \mathfrak{\Im} e+t\left(e^{*} \mathfrak{\Im} f\right) .
$$

Now $\mathfrak{B}^{\prime}$ has the form

$$
\mathfrak{B}^{\prime}=(e+f)^{*} \mathfrak{\Im}(e+f)
$$

since $(e+f)^{*} x(e+f)=e^{*} x e+\left(e^{*} x f+f^{*} x e\right)=e^{*} x e+t\left(e^{*} x f\right)$ using $f^{*} \mathfrak{f} f=0$. In particular, $\mathfrak{B}^{\prime}$ is automatically an inner ideal. We show that if $\mathfrak{B}$ is not a point space then $\mathfrak{B}=\mathfrak{B}^{\prime}$, while if $\mathfrak{B}$ is a point space so is $\mathfrak{B}^{\prime}$ and therefore by maximality $\mathfrak{B}=\mathfrak{B}^{\prime}$ again.

To show $\mathfrak{B}=\mathfrak{B}^{\prime}$ it suffices to show $\mathfrak{B}_{10}=\mathfrak{B}_{10}^{\prime}$, where $\mathfrak{B}_{10}^{\prime}=e^{*} \mathfrak{B}^{\prime} e^{\prime}=e^{*} \mathfrak{\Im} f$. Any transformation $x_{10}=e^{*} x f \in e^{*} \Im f$ vanishes on $\operatorname{Ker} f=\operatorname{Ker} c_{10}$, as do all $c_{10}^{\prime} \in e^{*} \mathfrak{B} e^{\prime}$, so it suffices to find $c_{10}^{\prime} \in \mathfrak{B}_{10}$ with $c_{10}^{\prime}(v)=x_{10}(v)$ (recall $c_{10}(v)=v_{0}$ so $\mathfrak{B}=\Phi v$ $+\operatorname{Ker} c_{10}$ ) since then $c_{10}^{\prime}=x_{10}$ on all of $\mathfrak{B}$. As $x_{10}(v) \in e^{* \mathfrak{B}}$ it is enough if $e^{*} \mathfrak{B} e^{\prime}(\mathfrak{B})$ $=e^{*}(\mathfrak{B})$. For any $y, z \in \mathfrak{J}$ we have $c^{\prime}=\left\{e^{*}\right.$ ye $\left.z c\right\} \in\{\mathfrak{B} \mathfrak{I} \mathfrak{B}\} \subset \mathfrak{B}$, and $c_{10}^{\prime}(v)$ $=\left(e^{*} y e\right) z c_{10}(v)=e^{*} y e z\left(v_{0}\right)$ because $e e^{\prime}=e^{*} c e^{\prime}=0$. Thus we hope $e^{*}(\mathfrak{B})=e^{*} \Im e \Im\left(v_{0}\right)$ $=e^{*} \mathfrak{\Im} e\left(v_{0}^{\perp}\right)$. By the lemma $e^{*} \mathfrak{g} e\left(v_{0}^{\perp}\right)$ will be all of $e^{*}(\mathfrak{B})$ unless $e\left(v_{0}^{\perp}\right)$ is 1-dimensional, $e\left(v_{0}^{\perp}\right)=\Phi u_{0}$, in which case $e^{*} \Im e\left(v_{0}^{\perp}\right)=e^{*} \Im u_{0}=e^{*}\left(u_{0}^{\perp}\right)$. Now $w \in u_{0}^{\perp} \Leftrightarrow e^{*} w \in v_{0}^{\perp \perp}$ $=\Phi v_{0}$, so $u_{0}^{1}=e^{* *}(\mathfrak{B})+\Phi v_{0}$. Thus $\left(e^{*} \mathfrak{\jmath} e\right) u_{0}=e^{*}\left\{e^{\prime *}(\mathfrak{B})+\Phi v_{0}\right\}=\Phi v_{0}$. Note $b\left(u_{0}\right)$ $=\lambda v_{0}$ where $\lambda \neq 0$ since $u_{0}=e\left(u_{0}\right)=d b\left(u_{0}\right)$. For any $y=e^{*} y e \in e^{*} \Im e$ we may (by subtracting off a suitable multiple of $b$ ) assume $y\left(u_{0}\right)=0$. But then $y(\mathfrak{B}) \subset u_{0}^{\perp}$, $y(\mathfrak{B})=e^{*} y(\mathfrak{B}) \subset e^{*}\left(u_{0}^{\perp}\right)=\Phi v_{0}$, while we saw that if $y$ has rank 1 then $y \Im y=y^{*} \Im y=0$ and by regularity $y=0$. Thus $y \in e^{*} \Im e$ implies $y \in \Phi b$, so that if $b \Im b \neq \Phi b$ we must have $e^{*} \mathfrak{J} e \mathfrak{}\left(v_{0}\right)=e^{*}(\mathfrak{B})$ and $\mathfrak{B}=\mathfrak{B}^{\prime}$.

Now consider what happens if $\mathfrak{B}$ is a maximal point space, $b \Im b=\Phi b$. Any element of $\mathfrak{B}^{\prime}$ may be written as $b^{\prime}=\lambda b+t\left(e^{*} x f\right)$ for some $\lambda \in \Phi$ and $x \in \mathfrak{F}$. If $y \in \mathfrak{F}$ then $b y b=\alpha b$ for some $\alpha$, hence $b y e^{*}=b y b d=\alpha b d=\alpha e^{*}$; also $e^{*} x f y b$ $=b\{d x f y\} b \in b \mathfrak{A} b$ implies $t\left(e^{*} x f y b\right)=\beta b$ for some $\beta$, hence

$$
e^{*} x f y e^{*} x f=\left\{e^{*} x f y b+b y f^{*} x e\right\} d x f
$$

(recall $\left.f^{*} \Im f=0\right)=\beta b d x f=\beta e^{*} x f$. If we combine these and note at $(z)+t(z) a^{*}$ $=t(a z)+t\left(z a^{*}\right)$ we get

$$
\begin{aligned}
b^{\prime} y b^{\prime} & =\lambda^{2} b y b+\lambda t\left(b y e^{*} x f\right)+\lambda t\left(e^{*} x f y b\right)+t\left(e^{*} x f y e^{*} x f\right) \\
& =\lambda^{2} \alpha b+\lambda \alpha t\left(e^{*} x f\right)+\lambda \beta b+\beta t\left(e^{*} x f\right) \\
& =\{\lambda \alpha+\beta\} b^{\prime}
\end{aligned}
$$

so that $b^{\prime}$ is a point and $\mathfrak{B}^{\prime}$ a point space. 
The remaining case is where all $c_{10}^{\prime}$ have the same range, Range $c_{10}^{\prime}=$ Range $f$ $=\Phi v_{0}$ for $f=c_{10} w$ ( $w$ as before). Here again $f$ is an idempotent of rank 1, but now $e^{*} f=f e^{*}=f$. In this case $c_{10}^{\prime}=f c_{10}^{\prime}$, so

$$
\mathfrak{B} \subset \mathfrak{B}^{\prime}=e^{*} \mathfrak{\jmath} e+t\left(f \Im e^{\prime}\right) .
$$

We claim necessarily $\mathfrak{B}$ is a point space in this case, $b \Im b=\Phi b$ : we saw $e^{*} \mathfrak{J} e \Im\left(v_{0}\right) \subset \mathfrak{B}_{10}(v) \subset f \Im e^{\prime}(v)=\Phi v_{0}$, and $e^{*} \mathfrak{J} \mathfrak{J}\left(v_{0}\right)=e^{*}(\mathfrak{B})\left(\neq \Phi v_{0}\right.$ since $e$ has rank $>1$ ) unless $b \Im b=\Phi b$. Thus $\mathfrak{B}$ is a point space. We claim $\mathfrak{B}^{\prime}$ is too, whence $\mathfrak{B}=\mathfrak{B}^{\prime}$. Again writing $b^{\prime}=\lambda b+t\left(f x e^{\prime}\right)$ we argue as before to get $b^{\prime} y b^{\prime}=\{\lambda \alpha+\beta\} b^{\prime}$ for any $y \in \Im$ where $b y b=\alpha b$ (hence $b y f=b y e^{*} f=b y b d f=\alpha f$ ) and $t\left(f x e^{\prime} y b\right)=\beta b$ (hence $\left.f x e^{\prime} y f=\beta f\right)$.

6. The algebras $\Im(Q, c)$. For algebras determined by quadratic forms, degenerate or not, we have the easy

THEOREM 6. The inner ideals in a Jordan algebra $\Im(Q, c)$ for a quadratic form $Q$ on a vector space $\mathfrak{X}$ over a field are precisely the totally isotropic subspaces of $\mathfrak{X}$ (together with $\mathfrak{X}$ itself).

Proof. If $\mathfrak{B}$ is a totally isotropic subspace of $\mathfrak{X}$ then $U_{\mathfrak{B} \Im} \mathfrak{\Im}=Q\left(\mathfrak{B}, \mathfrak{\Im}^{*}\right) \mathfrak{B}-Q(\mathfrak{B}) \mathfrak{\Im}^{*}$ $=Q\left(\mathfrak{B}, \mathfrak{J}^{*}\right) \mathfrak{B} \subset \mathfrak{B}$ and $\mathfrak{B}$ is an inner ideal.

Conversely, if $\mathfrak{B}$ is a proper inner ideal it contains no invertible elements; since $x \in \mathfrak{\Im}(Q, c)$ is invertible if and only if $Q(x) \neq 0$, we see $Q(\mathfrak{B})=0$ and $\mathfrak{B}$ is totally isotropic.

If $Q$ is nondegenerate then $\Im(Q, c)$ is regular : clearly $x \in U_{x} \mathfrak{\Im}=\mathfrak{\Im}$ if $x$ is invertible, and if $Q(x)=0$ then $Q(x, \mathfrak{\Im}) \neq 0$ by nondegeneracy so $Q(x, y)=1$ for some $y$ and $U_{x} y^{*}=Q(x, y) x=x$. Thus the principal inner ideal $U_{x} \mathfrak{J}$ is either $\mathfrak{J}$ or $\Phi x$.

COROllary. If $Q$ is nondegenerate then $\Im(Q, c)$ has the a.c.c. and d.c.c. on principal inner ideals. It has the a.c.c. and d.c.c. on all inner ideals if and only if there are no infinite-dimensional totally isotropic subspaces.

To see the last statement, if $\mathfrak{B}$ is infinite dimensional and totally isotropic with basis $x_{1}, x_{2}, \ldots$ then the $\mathfrak{B}_{i}^{\prime}=\left\{x_{i}, x_{i+1}, \ldots\right\}$ form a properly descending sequence of inner ideals and the $\mathfrak{B}_{i}^{\prime \prime}=\left\{x_{1}, \ldots, x_{i}\right\}$ a proper increasing sequence. Conversely, if the $\mathfrak{B}_{i}$ form a properly increasing (or decreasing) sequence of inner ideals the dimensions must be increasing (or decreasing), so $\mathfrak{B}=\bigcup \mathfrak{B}_{\mathfrak{i}}$ (or $\mathfrak{B}=\mathfrak{B}_{1}$ ) must be infinite dimensional and isotropic.

In characteristic 2 there are some slight variants on the algebras $\Im(Q, c)$ which are obtained (up to isotopy) as follows: inside a split $\Im(Q, c)=\Phi e_{1}+\mathfrak{X}_{0}+\Phi e_{2}$ for

$$
Q\left(\alpha e_{1}+x_{0}+\beta e_{2}\right)=\alpha \beta-Q_{0}\left(x_{0}\right)
$$

where $Q_{0}$ is nondegenerate, consider the ample outer ideal

$$
\Im\left(Q_{0}, \Phi_{0}\right)=\Phi_{0} e_{1}+\mathfrak{X}_{0}+\Phi_{0} e_{2}
$$


where $\Phi_{0}$ is an additive subgroup of $\Phi$ satisfying (i) $1 \in \Phi_{0}$, (ii) $\Phi^{2} \Phi_{0} \subset \Phi_{0}$, (iii) $Q_{0}\left(\mathfrak{t}_{0}\right) \Phi_{0} \subset \Phi_{0}$. (Thus we obtain $\Im\left(Q_{0}, \Phi_{0}\right)$ by restricting the "diagonal" entries to lie in an "ample" subspace $\Phi_{0}$ of $\Phi$.) If the characteristic is not 2 or $\Phi$ is perfect (so $\Phi^{2}=\Phi$ ) or if $Q_{0}\left(x_{0}, y_{0}\right)$ is not identically zero (so $Q_{0}\left(\mathfrak{X}_{0}\right) \supset Q_{0}\left(\mathfrak{X}_{0}, \Phi \mathfrak{X}_{0}\right)=\Phi$ ) we necessarily have $\Phi_{0}=\Phi$ and $\Im\left(Q_{0}, \Phi_{0}\right)=\Im(Q, c)$. Thus we consider only the case where $Q_{0}\left(\mathfrak{x}_{0}, \mathfrak{X}_{0}\right)=0$ and where $Q_{0}$ is still nondegenerate (so $Q_{0}\left(x_{0}\right)=0$ implies $\left.x_{0}=0\right)$. Note that this means $\mathfrak{\Im}_{0}=\mathfrak{I}\left(Q_{0}, \Phi_{0}\right)$ is "almost" a division algebra. Thus we should not be too surprised if $\Im_{0}$ contains few inner ideals.

THEOREM 7. The only proper inner ideals in the algebra $\Im_{0}=\Im\left(Q_{0}, \Phi_{0}\right)$ determined by a nondegenerate quadratic form $Q_{0}$ on $\mathfrak{X}_{0}$ with $Q_{0}\left(\mathfrak{X}_{0}, \mathfrak{X}_{0}\right)=0$ are the spaces $\Phi_{0} e_{1}, \Phi_{0} e_{2}$, and $\Phi_{0}\left(e_{1}+\lambda e_{2}+x_{0}\right)$ for $x_{0} \in \mathfrak{X}_{0}$ with $Q_{0}\left(x_{0}\right)=\lambda \neq 0$.

Proof. Again $x$ is invertible in $\Im_{0}$ if and only if $Q(x) \neq 0$ because the inverse $Q(x)^{-1} x^{*}=Q(x)^{-1}\left\{\alpha_{2} e_{1}-x_{0}+\alpha_{1} e_{1}\right\}$ of $x=\alpha_{1} e_{1}+x_{0}+\alpha_{2} e_{2}$ again belongs to $\Im_{0}$ (note $Q(x)^{-1} \alpha_{i}=Q(x)^{-2} Q(x) \alpha_{i}$ for $Q(x)^{-2} \in \Phi^{2}$, and $Q(x) \alpha_{i}=\alpha_{1} \alpha_{2} \alpha_{i}-Q_{0}\left(x_{0}\right) \alpha_{i}$ where $\alpha_{i}^{2} \alpha_{j} \in \Phi^{2} \alpha_{j} \subset \Phi_{0}$ and $\left.Q_{0}\left(\mathfrak{X}_{0}\right) \Phi_{0} \subset \Phi_{0}\right)$. Therefore any proper inner ideal is totally isotropic. Furthermore, $\Im_{0}$ is again regular, so any inner ideal is invariant under $\Phi_{0} \subset \Gamma\left(\Im_{0}\right)$.

If $Q(x)=0$ then $\alpha_{1} \alpha_{2}=Q_{0}\left(x_{0}\right)$. If $\alpha_{1}$ or $\alpha_{2}$ is zero then $Q_{0}\left(x_{0}\right)$ is too, whence $x_{0}=0$, and $x=\alpha_{1} e_{1}$ or $\alpha_{2} e_{2}$. Otherwise $\alpha_{1} \alpha_{2}=Q_{0}\left(x_{0}\right) \neq 0, x=\alpha_{1}\left(e_{1}+\lambda e_{2}+y_{0}\right)=\alpha_{1} y$ for $\lambda=\alpha_{1}^{-1} \alpha_{2}=\alpha_{1}^{-2} Q_{0}\left(x_{0}\right) \in \Phi_{0}$ and $y_{0}=\alpha_{1}^{-1} x_{0} \in \mathfrak{X}_{0}$. If $x$ belongs to an inner ideal $\mathfrak{B}$ so does $y$; any other element $x^{\prime}$ has (after subtraction of a suitable multiple of $y$ ) coefficient $\alpha_{1}^{\prime}=0$, hence $x^{\prime}=\alpha_{2}^{\prime} e_{2}$; but then $Q\left(y, x^{\prime}\right)=0$ implies $\lambda \alpha_{2}^{\prime}=0$, so $\alpha_{2}^{\prime}=0$ and $x^{\prime}=0$ and $\mathfrak{B}=\Phi_{0} y$.

Since all proper inner ideals are atoms in the lattice of inner ideals we have a

COROLlARY. The Jordan algebra $\Im\left(Q_{0}, \Phi_{0}\right)$ determined by a nondegenerate quadratic form $Q_{0}$ on $\mathfrak{X}_{0}$ with $Q_{0}\left(\mathfrak{X}_{0}, \mathfrak{X}_{0}\right)=0$ has a.c.c. and d.c.c. on all inner ideals. Any proper chain of inner ideals $\mathfrak{\Im}=\mathfrak{B}_{1}>\mathfrak{B}_{2}>\cdots>\mathfrak{B}_{r}>0$ has length $r \leqq 2$.

7. The algebras $\mathfrak{I}(N, c)$. The exceptional algebras $\mathfrak{S}_{\mathfrak{C}}\left(\mathfrak{S}_{3}, \gamma\right)$ can be obtained via the Springer Construction [6], wherein one starts with a cubic form $N$ on a vector space $\mathfrak{X}$ over a field $\Phi$ and a basepoint $c \in \mathfrak{X}$ where $N(c)=1$, and builds a Jordan algebra $\Im(N, c)$ with unit $c$ and $U$-operator

$$
U_{x} y=T(x, y) x-x^{\#} \times y .
$$

Here it is assumed that the symmetric bilinear form $T(x, y)=-\left.\partial_{x} \partial_{y} \log N\right|_{c}$ is nondegenerate, and that the adjoint mapping $x^{\#}$ defined by $T\left(x^{\#}, y\right)=\left.\partial_{y} N\right|_{x}$ satisfies the adjoint formula

$$
x^{\# \#}=N(x) x .
$$




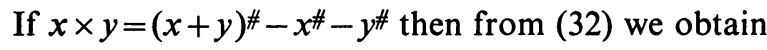

$$
\begin{gathered}
x^{\#} \times y^{\#}+(x \times y)^{\#}=T\left(x^{\#}, y\right) y+T\left(y^{\#}, x\right) x, \\
x^{\#} \times(x \times y)=N(x) y+T\left(x^{\#}, y\right) x .
\end{gathered}
$$

An element $x$ is invertible in $\Im(N, c)$ if and only if $N(x) \neq 0$.

Proposition 7. $\Im(N, c)$ is regular.

Proof. If $N(x) \neq 0$ then $x$ is invertible and $x \in U_{x} \mathfrak{F}=\mathfrak{J}$, so assume $N(x)=0$. If $x \neq 0$ but $x^{\#}=0$ then $T(x, y)=1$ for some $y$ by nondegeneracy, whence $U_{x} y=x$ by (31), while if $x^{\#} \neq 0$ then again $T\left(x^{\#}, y\right)=1$ for some $y$ and by (34) $x^{\#} \times(x \times y)=x$, whence $U_{x}(x \times y)=T(x, x \times y) x-x=T(x \times x, y) x-x=2 T\left(x^{\#}, y\right) x-x=x$ by (31).

The formula (31) shows that a subspace $\mathfrak{B}$ is an inner ideal in $\mathfrak{\Im}$ if and only if

$$
\mathfrak{B}^{\#} \times \mathfrak{\Im} \subset \mathfrak{B} .
$$

In particular, any subspace $\mathfrak{B}$ with $\mathfrak{B}^{\#}=0$ is an inner ideal, even a point space. If $\mathfrak{B}^{\#} \neq 0$ there is an element $b \in \mathfrak{B}$ with $d=b^{\#} \neq 0$; if $\mathfrak{B}$ is proper it contains no invertible elements, so $N(b)=0$ and hence $d^{\#}=b^{\# \#}=0$ by (32). But whenever $d^{\#}=0$ the subspace $d \times \mathfrak{I}$ is an inner ideal: by (33) any of its elements $d \times y$ has $(d \times y)^{\#}$ $=T\left(y^{\#}, d\right) d$, so $(d \times y)^{\#} \times \mathfrak{F}=T\left(y^{\#}, d\right) d \times \mathfrak{F} \subset d \times \mathfrak{\Im}$. By (35) this means $d \times \mathfrak{I}$ is inner. In our original situation, $d=b^{\#}$ and $\mathfrak{B} \supset b^{\#} \times \mathfrak{F}$ where the latter is an inner ideal. Indeed, when $b^{\#} \neq 0$ but $N(b)=0$ we claim $b^{\#} \times \mathfrak{\Im}$ is just the principal inner ideal $U_{b} \mathfrak{F}$. By regularity, $U_{b} \mathfrak{\Im}$ contains $\Phi b$ and hence $b^{\#} \times \mathfrak{I}$ by (31), while conversely we saw above that $b^{\#} \times \Im$ contains $b^{\#} \times(b \times y)=b$ for some $y$ and so (by (31)) all of $U_{b} \Im$. (We remark that if $b^{\#}=0$ the principal inner ideal determined by $b$ is $U_{b} \Im=\Phi b$, while if $N(b) \neq 0 U_{b} \Im=\Im$.)

We now specialize to the case $\mathfrak{S}(N, c)=\mathfrak{S}_{\mathcal{C}}\left(\mathfrak{夭}_{3}, \gamma\right)$ for $\mathfrak{S}$ a Cayley algebra. We have already characterized the inner ideals in the Main Theorem, but we give another derivation of these results based on geometric reasoning (see [1]). We need the fact that the structure group of $\mathfrak{\Im}=\mathfrak{S}\left(\mathfrak{C}_{3}, \gamma\right)$ acts transitively on points, an element being a point if $x^{\#}=0$ but $x \neq 0$.

THEOREM 8. A subspace $\mathfrak{B}$ of $\mathfrak{S}=\mathfrak{S}\left(\mathfrak{C}_{3}, \gamma\right)$ is an inner ideal if and only if it is one of three types: (i) $\mathfrak{B}=\mathfrak{I}$, (ii) $\mathfrak{B}=b^{\#} \times \mathfrak{I}$ for any $b \in \mathfrak{B}$ with $b^{\#} \neq 0$, (iii) a point space $\mathfrak{B}^{\#}=0$. If $\mathfrak{B}=\mathfrak{I}$ then $\mathfrak{B}=U_{1} \mathfrak{\Im}$ is principal. If $\mathfrak{B}=b^{\#} \times \mathfrak{\Im}$ then $\mathfrak{B}=U_{b} \mathfrak{\Im}$ is principal. If $\mathfrak{C}$ is a division algebra then the only point spaces are the spaces $\Phi b$, and in this case $\mathfrak{B}=U_{b} \Im$ is principal.

Proof. For arbitrary $\Im(N, c)$ we have noted that the three types are all inner ideals. Conversely, if $\mathfrak{B} \neq \mathfrak{J}$ then $N(\mathfrak{B})=0$; in this case either $\mathfrak{B}^{\#}=0$ or $b^{\sharp} \neq 0$ for some $b \in \mathfrak{B}$, in which case we saw $\mathfrak{B} \supset b^{\#} \times \mathfrak{I}=U_{b} \mathfrak{s}$. We will show that for $\mathfrak{\Im}=\mathfrak{S}_{(}\left(\mathfrak{E}_{3}, \gamma\right)$ such a $b^{\#} \times \mathfrak{\Im}$ is maximal proper, hence $\mathfrak{B}=b^{\#} \times \mathfrak{\Im}$. Now $b^{\#} \times \mathfrak{\Im}$ will be maximal if $S^{*}\left(b^{\#} \times \Im\right)=S\left(b^{\#}\right) \times \Im$ is maximal for some $S$ in the structure group. 
Since $b^{\#}$ is a point, by transitivity we can choose $S$ so that $S\left(b^{\#}\right)=e_{1}$. Thus we may assume $b^{\#}$ is the diagonal idempotent $e_{1}$ in $\mathfrak{S}_{(}\left(\mathfrak{S}_{3}, \gamma\right), b^{\#} \times \mathfrak{J}=\mathfrak{\Im}_{0}\left(e_{1}\right)$. If $\mathfrak{B}>\mathfrak{J}_{0}\left(e_{1}\right)$, say some $x=\alpha e_{1}+c[12]+d[13] \neq 0$ belongs to $\mathfrak{B}$, then $x \neq \alpha e_{1}$ (since $1=e_{1}+e_{2}+e_{3}$ $\notin \mathfrak{B})$, so $c$ or $d$ is nonzero. Now $U_{x, \mathfrak{C}[23]} e_{2}=\left\{c[12] e_{2} \mathfrak{E}[23]\right\}=c \mathfrak{C}[13] \subset U_{\mathfrak{B} \mathfrak{I}} \subset \mathfrak{B}$ and similarly $d \mathfrak{C}[12] \subset \mathfrak{B}$. Thus $\mathfrak{B} \supset \mathfrak{D}_{2}[12]+\mathfrak{D}_{3}[13]$ for subspaces $\mathfrak{D}_{i} \subset \mathfrak{C}$ with $\mathfrak{D}_{i} \mathfrak{C} \subset \mathfrak{D}_{j}$. Then $\mathfrak{D}_{3}=\mathfrak{D}_{2}=\mathfrak{D}$ is a right ideal, so $\mathfrak{D}=0$ or $\mathfrak{C}$; since $x \neq 0$ we must have $\mathfrak{D}=\mathfrak{C}$, $1[12] \in \mathfrak{B}$, consequently $\mathfrak{B}$ contains the invertible element $1[12]+e_{3}$, a contradiction. Thus $e_{1} \times \mathfrak{I}$ is maximal, hence $d \times \mathfrak{\Im}$ is maximal for any point $d$.

Finally, if $\mathfrak{C}$ is a division algebra we must show any point space has dimension 1 . If $b \neq 0$ lies in a point space $\mathfrak{B}$ we may assume (applying an element of the structure group) that $b=e_{1}$. Then $b \times \mathfrak{B}=0$, and since $e_{1} \times e_{i}=e_{j}, e_{1} \times a[23]=-a[23]$ with all other product zero we see any other $b^{\prime} \in \mathfrak{B}$ has $b^{\prime}=\alpha_{1} e_{1}+a_{2}[13]+a_{3}[12]$. But $b^{\prime \prime}=b^{\prime}-\alpha_{1} e_{1} \in \mathfrak{B}$ has $b^{\prime \prime 2}=n\left(a_{2}\right)\left(e_{1}+e_{3}\right)+n\left(a_{3}\right)\left(e_{1}+e_{2}\right)+\bar{a}_{3} a_{2}[23]$ so $n\left(a_{2}\right)=n\left(a_{3}\right)$ $=0$. In a division algebra this implies $a_{2}=a_{3}=0, b^{\prime} \in \Phi b$, and $\mathfrak{B}=\Phi b$.

The point spaces of the split $\mathfrak{S}_{(}\left(\mathfrak{C}_{3}, \gamma\right)$ have been investigated by T. A. Springer and F. Veldkamp [7, Proposition 3.14]. By geometric methods they obtained the classification which we arrived at in the Main Theorem; their work assumed characteristic $\neq 2$, but J. Faulkner has shown [1] how the geometry may be treated uniformly in all characteristics. The lattice of inner ideals seems to play an important role in geometries associated with these algebras.

\section{REFERENCES}

1. J. Faulkner, Octonion planes defined by quadratic Jordan algebras, Mem. Amer. Math. Soc. No. 104 (1970).

2. N. Jacobson, Structure theory of quadratic Jordan algebras, Lecture Notes, Tata Institute, Bombay, 1970.

3. K. McCrimmon, A general theory of Jordan rings, Proc. Nat. Acad. Sci. U.S.A. 56 (1966), 1072-1079. MR 34 \#2643.

4. - The radical of a Jordan algebra, Proc. Nat. Acad. Sci. U.S.A. 59 (1969), 671-678.

5. - Quadratic Jordan algebras and cubing operations, Trans. Amer. Math. Soc. 153 (1971), 265-278.

6. - The Freudenthal-Springer-Tits constructions of exceptional Jordan algebras, Trans. Amer. Math. Soc. 139 (1969), 495-510. MR 39 \#276.

7. - Homotopes of alternative algebras, Math. Ann. 191 (1971), 253-262.

8. T. A. Springer and F. van der Blij, Octaves and triality, Nieuw Arch. Wisk. (3) 8 (1960), 158-169. MR 23 \#A947.

9. T. A. Springer and F. Veldkamp, On Hjelmslev-Moufang planes, Math. Z. 107 (1968), 249-263. MR 40 \#1884.

UNIVERSITY OF VIRGINIA, Charlottesville, Virginia 22904 